\title{
NOISE AND COMPETITION IN STRATEGIC OLIGOPOLY ${ }^{*}$
}

\author{
by \\ Ramdan Dridi \\ London School of Economics and Political Science \\ and \\ Laurent Germain \\ London Business School and Ecole Supérieure de Commerce de Toulouse
}

Contents:
Abstract
1. Introduction
2. The Setup
3. Equilibrium Existence and Uniqueness
4. Equilibrium Properties
5. Symmetric Case
6. Concluding Remarks
References
Appendices

Discussion Paper

No. EM/00/395

June 2000
The Suntory Centre

Suntory and Toyota International Centres for Economics and Related Disciplines London School of Economics and Political Science Houghton Street London WC2A 2AE Tel.: 02074057686

* We are particularly indebted to Bruno Biais, Gilles Chemla, Antoine Faure-Grimaud, Mike Fishman, Jean-Claud Gabillon, André Grimaud, Thomas Mariotti, Jean-Claud Rochet, David Stolin and seminar participants at Toulouse for long conversations and very thoughtful comments which have greatly improved the quality of this paper. All remaining errors are our own. 


\begin{abstract}
Focusing on homogeneous beliefs, we can distinguish two commonly shared ideas that, i) the competition between informed traders destroys their trading profits, ii) trading with a noisy signal brings about a loss in the expected profits. So far, it has been proved in the latter framework, that when $\mathrm{N}$ strategic and perfectly informed traders compete in the financial market, i) the informativeness of prices increases with the degree of competition and, ii) the aggregate and individual profits go to 0 when $\mathrm{N}$ is large. In this paper, we propose a general study where $\mathrm{N}$ strategic informaed agents have heterogeneous beliefs, i.e. are endowed with noisy information and compete à la Nash. We prove the existence and uniqueness of a linear equilibrium generalizing Kyle (1985) results to the case of $\mathrm{N}$ informed traders when the insiders have heterogeneous beliefs. In this general framework, we derive the following striking results: for certain regions of noise and numbers of competitors in excess of four, i) each individual expected profit is greater than the one obtained in the perfectly informed (and homogeneous beliefs) case; ii) the aggregate profit has a finite (strictly) positive limit when $\mathrm{N}$ is large. iii) Even when an infinite number of insiders compete in the market, the price is no longer efficient and does not fully reveal the final liquidation value of the risky asset. iv) In the particular case where each informed agent is endowed with a signal the precision of which is the same, a) we show that there exists an optimal level of noise for which each individual expected profit is maximized; b) we show that there exists an optimal size of the market for which the aggregate expectged profit is maximized; c) the liquidity is an increasing function of the number of informed traders but has a finite limit for large $\mathrm{N}$; d) the informativeness of prices is a decreasing function of the number of informed traders.
\end{abstract}

Keywords: Competition; optimal noise; price manipulation.

JEL Nos.: D43, D82, G14, G24.

(C) by the authors. All rights reserved. Short sections of text, not to exceed two paragraphs, may be quoted without explicit permission provided that full credit, including (C) notice, is given to the source. 


\section{Introduction}

How much information is reflected into prices? How do prices reflect and aggregate information? In the case of competitive traders, Grossman (1976) shows that the equilibrium price aggregates the dispersed private information. If there is no noise in the market - or more generally no sources of uncertainties - prices fully reveal the private information dispersed among the traders (Grossman and Stiglitz (1980)). Those results are mitigated when considering the case of strategic traders who are aware of their impact into prices. In the case of a monopolistic perfectly informed risk neutral trader, Kyle (1985) shows that the price is not fully efficient and only half of the insider's private information is revealed at the equilibrium. The monopoly keeps his informational advantage by optimally camouflaging his information in the noise. Those results are different when there are many strategic traders who compete in the market. The competition diminishes the insiders informational advantage. Kyle (1984) and Admati and Pfleiderer (1988a) put forward that the informativeness of prices is an increasing function of the degree of competition between the traders. Indeed, prices are all the more informative than the competition between the traders is fierce. In particular, when a large number of strategic perfectly informed traders compete in the market the price is strongly efficient. In the same line but in a dynamic setting Holden and Subrahmanyam (1992-1995) shows that the speed of revelation of information increases with the number of competitors.

Another strand of the literature investigates the effects of noise onto prices. There are different sources of noise. The noise can stem from the presence of noise traders (Kyle (1985)) or other sources of uncertainties like endowment shocks when the agents are risk averse (Biais, Martimort and Rochet (1997)) or a random supply, Grossman and Stiglitz (1980). When the distribution of the risky asset is bimodal or has a three points support, Biais and Germain (1997) and Germain (1998) show that perfectly informed traders would be better off adding noise exante into prices if they can commit to do so. This is no longer the case when the distribution is normal and the pricing rule is linear like it is in the Kyle (1985) framework. Indeed, in the standard Kyle (1985) model we know that, the more precise is the available information

the better is the informed monopoly (see section 2 for more details). In other words, it is not optimal ex-ante to add noise into prices.

The first conclusion we can draw from those financial studies is that:

- On the one hand, the noise diminishes the informativeness of prices,

- and, on the other hand, the competition increases the informativeness of prices.

Could it be that any trade-off arise? Is the competition effect sufficiently strong that, whatever the level of noise in the market the competitors always bear the cost of noise? If so, for which level of noise in the market is it true? When the distribution of the risky asset is bimodal Germain (1998) shows how N strategic indirect sellers of information can commit to add the 
optimal ex-ante level of noise in the market. ${ }^{1}$ Are there optimal regions of noise in a more general framework also? To address those issues in the present paper, we propose the general study where $\mathrm{N}$ strategic informed agents who have heterogeneous beliefs i.e. are endowed with noisy information $\tilde{S}_{i}=\tilde{v}+\tilde{\varepsilon}_{i} i=1, \ldots, N$ and compete à la Nash. ${ }^{2}$ In the same vein, Foster and Viswanathan (1996) and Back (1995) have studied the impact of correlations on imperfect competition while focusing on heterogeneous beliefs. The main difficulty arising in this type of models is that agents have to "forecast the forecasts of others".

More precisely, there are two commonly shared ideas that i) the competition between informed traders destroys their expected trading profits ii) trading with a noisy signal brings about a loss in the expected profits.

So far, it is just proved that when $\mathrm{N}$ strategic traders have homogeneous beliefs ${ }^{3}$ and compete in the financial market i) the informativeness of prices increases with the degree of competition and ii) the aggregate and individual profits go to 0 when $\mathrm{N}$ is large (See Admati and Pfleiderer (1988a)). And as a matter of fact the same authors rightly notice, that "it is straightforward to show that with risk neutral traders, the total profits of the informed traders, $n \pi(n)$ are decreasing in $\mathrm{n}$. This is analogous to the result that industry profits are decreasing in the number of firms in a Cournot oligopoly".

We stress in this paper that, in the heterogeneous beliefs framework, strategic competition should be rather parameterized by an N-dimensional vector composed by the precision of each insider's signal. In effect, the classical Cournot result holds only for certain structure of information (in particular in the homogeneous beliefs setting). In the heterogeneous beliefs framework, we show that the competition can be mitigated by the presence of noise. ${ }^{4}$

Moreover we emphasize on the fact that two levels of heterogeneity have to be distinguished in our general financial study. Heterogeneous beliefs can stem in this set-up:

- either from different signals but the same distribution that is identically distributed, ${ }^{5}$

- or from different probability distributions.

We will proceed in this paper as follows: first we develop the theory in the latter generic case and particularize the results to the former case and referred to as the symmetric case.

More precisely, we derive the following striking results: for certain regions of noise and a number of competitors $N \geq 4$,

\footnotetext{
${ }^{1}$ The commitment is made credible through contracts for the sale of information.

${ }^{2}$ In the standard literature, the previous questions are often solved within the homogeneous beliefs paradigm. This is for instance the case of Admati and Pfleiderer (1988a-b), Foster and Viswanathan (1994) and Holden and Subrahmanyam (1992).

${ }^{3}$ The simplest and widespread example corresponds to the case where traders are perfectly informed.

${ }^{4}$ In a different set-up and a dynamic model Foster and Viswanathan (1996) show that the competition may depend on the correlation between the traders' signals. However the crucial assumption in their framework is that the signals aggregate to a sufficient statistic of the final liquidation $v: \frac{1}{N} \sum_{i=1}^{N} s_{i \circ}=v$.

${ }^{5}$ This corresponds to what has mainly been studied.
} 
- i) each individual expected profit is greater than the one obtained in the perfectly informed case;

- ii) the aggregate profit has a finite (strictly) positive limit when $\mathrm{N}$ is large.

- iii) Even when an infinite number of insiders compete in the market the price is no longer efficient and does not fully reveal the final value of liquidation of the risky asset.

- iv) In the particular case where each heterogeneous informed agent is endowed with a signal the precision of which is the same for each agent we show that there exists an optimal level of noise for which each individual profit is maximized.

- For each given level of precision of the signals, there exists an optimal size of the market for which the aggregate expected profit is maximized.

- vi) The liquidity is not always an increasing function of the number of informed traders and may have a finite limit for large $\mathrm{N}$.

- vii) The informativeness of prices is not always increasing with the number of informed traders.

Furthermore, we address another issue. In the framework of the linear equilibria modeling with normality and when the traders have heterogeneous beliefs two related issues remain unsolved. i) Do we still have the uniqueness of a particular linear equilibrium (linear in prices as well as in quantities)? ii) Can we extend this framework to the case where the informed traders are not necessarily endowed with the same signal?

As a matter of fact, Kyle (1984) and Admati and Pfleiderer (1988b) have studied the case where $\mathrm{N}$ informed traders endowed with the same signal $S$ compete in the market and have shown the existence of a linear equilibrium. Foster and Viswanathan (1996) and Vives (1995) have shown also the existence of a linear equilibrium when traders have heterogeneous beliefs. In this respect, our results are the following. We first show the existence and the uniqueness of a linear equilibrium. This generalizes the result derived by Kyle (1985) in the case of a single perfectly informed monopoly to the case where $\mathrm{N}$ strategic informed traders are endowed with different signals. This relies to the best of our knowledge on new proofs since so far what has been exhibited is the existence and not the uniqueness of the particular linear equilibrium. In effect, while in Kyle (1985) and Holden and Subrahmanyam (1992), each informed trader knows at the equilibrium the quantity submitted by each other one, in our framework there is still a persisting uncertainty due to the noisy signals and therefore the informed noisy trades. ${ }^{6}$

The paper is organized as follows. We first lay out in section 2 the general set up and the considered model generalizing Kyle (1985). We show in section 3 the existence and uniqueness of a linear equilibrium and characterize the equilibrium as well as the expected profits performed

\footnotetext{
${ }^{6}$ This uncertainty is reinforced in the general case where insiders signals are distributed according to non identical probability distributions.
} 
by each informed agent in the heterogeneous beliefs context. In section 4, we provide the general study of the equilibrium properties and delineate the regions for which each informed traders is better off with respect to the perfectly informed case. We discuss in section 5 the particular situation in which each informed heterogeneous agent is endowed with a signal of which precision is the same and show the existence of an optimal level of noise for each given number of insiders as well the optimal size of the market for each given level of precision. We then focus on the individual reaction, the liquidity, individual and aggregate profits and informativeness properties at equilibrium. Finally section 6 states some concluding remarks. 


\section{The setup}

We consider a financial market with a risky asset normally distributed $\widetilde{v} \leadsto \mathcal{N}\left(0, \sigma_{v}^{2}\right)$. We denote the final liquidation value $\mathrm{v}$. There are three types of agents:

1. $N$ risk neutral informed traders who observe in advance a signal $\widetilde{S}_{i}=\widetilde{v}+\widetilde{\varepsilon}_{i}$, where $\widetilde{\varepsilon}_{i}$ is a random disturbance term (the noise) and we will assume that:

$$
\begin{aligned}
& \widetilde{\varepsilon}_{i} \leadsto \mathcal{N}\left(0, \sigma_{i}^{2}\right) \\
& \left\{\widetilde{v}, \widetilde{\varepsilon}_{1}, \ldots, \widetilde{\varepsilon}_{N}\right\} \text { are mutually independent. }
\end{aligned}
$$

2. Liquidity traders who submit market orders $\widetilde{u} \leadsto \mathcal{N}\left(0, \sigma_{u}^{2}\right)$ and such that $\left\{\widetilde{v}, \widetilde{\varepsilon}_{1}, \ldots, \widetilde{\varepsilon}_{N}, \widetilde{u}\right\}$ are mutually independent.

3. Risk neutral market makers, who observe the aggregate volume $\widetilde{w}$ and set rationally the price in a Bayesian way.

A strategy for the informed agent $i=1, \ldots, N$ is a Lebesgue measurable function: $X_{i}: \mathbb{R} \longrightarrow$ $\mathbb{R}$, determining his market order as a function of the observed signal $S_{i}$. For given strategies $\left(X_{1}, \ldots, X_{N}\right)$, let $\widetilde{x}_{i}=X_{i}\left(\widetilde{S}_{i}\right)$. These strategies determine the aggregate order flow $\widetilde{w}=\sum_{i=1}^{N} \widetilde{x}_{i}+$ $\widetilde{u}$.

Market makers observe the realization of the order flow, but not any of its components, and engage in a competitive auction. The outcome of this competition is described by a Lebesgue measurable function: $P: \mathbb{R} \longrightarrow \mathbb{R}$.

Given $\left(P, X_{1}, \ldots, X_{N}\right)$, we denote $\widetilde{p}=P(\widetilde{w})$ and let $\widetilde{\pi}_{i}=(\widetilde{v}-\widetilde{p}) \widetilde{x}_{i}$ the resulting trading profit of each insider $i=1, \ldots, N$.

The equilibrium conditions are that the competition in which market makers are involved drives their expected profits to zero conditional on the aggregate submitted volume and that the informed traders choose their trading strategies so as to maximize their expected profits.

Definition 2.1 : $\left(P, X_{1}, \ldots, X_{N}\right) \in L_{2}^{N+1}$ is an equilibrium if: ${ }^{7}$

$$
E[\widetilde{v}-\widetilde{p} / \widetilde{w}]=0
$$

and for all $X_{i} \in L_{2}$, given the (rational) beliefs of the market makers, and the corresponding price function $(P()$.$) , each informed chooses X_{i}$ to maximize his expected profits:

$$
\widetilde{x}_{i} \in \underset{x \in \mathbb{R}}{\operatorname{Argmax}} E\left[\left(\widetilde{v}-P\left(x+\sum_{j \neq i} \widetilde{x}_{j}+\widetilde{u}\right)\right) x / S_{i}\right] .
$$

\footnotetext{
${ }^{7} L_{2}$ corresponds to the set of square integrable Lebesgue measurable functions.
} 
Definition 2.2 $:\left(P, X_{1}, \ldots, X_{N}\right) \in L_{2}^{N+1}$ is a linear equilibrium if in addition there exists a scalar $\lambda \in \mathbb{R}_{+}$:

$$
\widetilde{p}=E[\widetilde{v} / \widetilde{w}]=\lambda \widetilde{\omega}
$$

We derive the unique perfect Bayesian linear equilibrium of this game. 


\section{Equilibrium Existence and Uniqueness}

We first start by stating the result in the monopoly case which is distinguished from the oligopoly case $(N \geq 2)$. We characterize the linear equilibrium in the case of a monopolistic imperfectly informed trader who observes a signal $\widetilde{S}=\widetilde{v}+\widetilde{\varepsilon}$. This signal corresponds to the previous oligopoly description but here $\mathrm{N}=1$.

Proposition 3.1 : In the monopoly case $(N=1)$, there exists a unique linear equilibrium defined by $\widetilde{x}=\beta^{*}(\sigma) \widetilde{S}$ and $\widetilde{p}=\lambda^{*}(\sigma) \widetilde{\omega}$, where $\sigma^{2}=\operatorname{Var}(\widetilde{\varepsilon})$, such that:

$$
\begin{aligned}
& \beta^{*}(\sigma)=\frac{\sigma_{u}}{\sigma_{v}} \frac{1}{\sqrt{1+\tau}}, \\
& \frac{1}{\lambda^{*}(\sigma)}=2 \frac{\sigma_{u}}{\sigma_{v}} \sqrt{1+\tau} \\
& \tau=\frac{\sigma^{2}}{\sigma_{v}^{2}} .
\end{aligned}
$$

Proof : See appendix A.1.

Figure 1 represents the individual monopolistic reaction as a function of the noise and where the normalization is taken with respect to the perfectly informed case. We observe that the more accurate the information is and the more reactive is the monopoly.

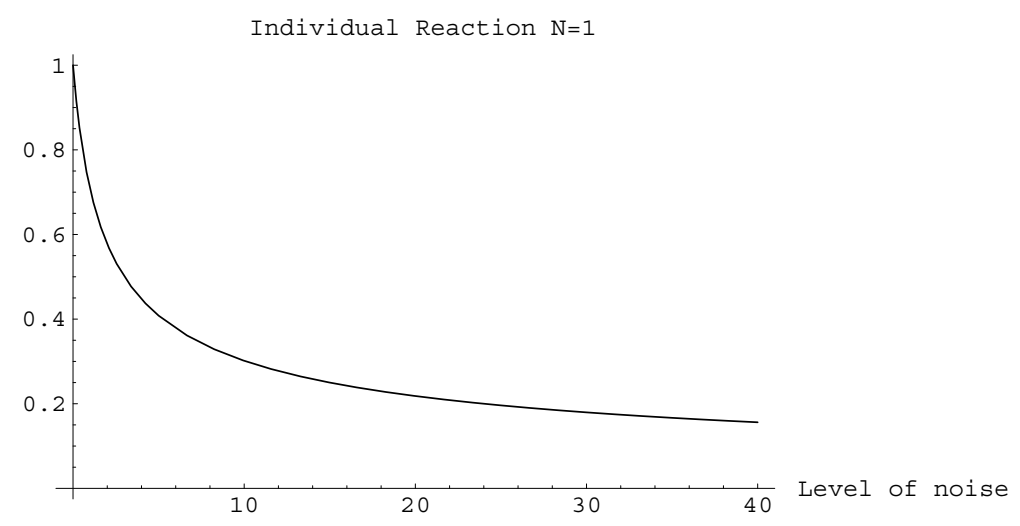

Figure 1: Individual Reaction $(\mathrm{N}=1)$.

Figure 2 represents the liquidity in the monopolistic case as a function of the noise and where the normalization is taken with respect to the perfectly informed case. We observe that the more accurate the information is and the less liquid the market is. 


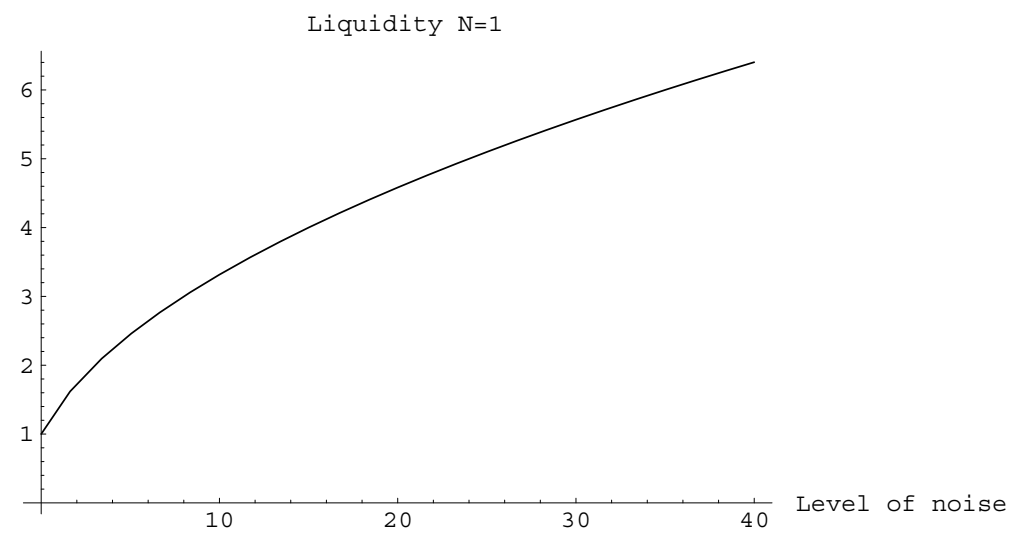

Figure 2: Liquidity $(\mathrm{N}=1)$.

Proposition 3.2 : In the monopoly case $(N=1)$, the expected profit $\pi^{*}(\sigma)$ is at equilibrium given by:

$$
\begin{aligned}
& \pi^{*}(\sigma)=\frac{1}{2} \sigma_{u} \sigma_{v} \frac{1}{\sqrt{1+\tau}} \\
& \tau=\frac{\sigma^{2}}{\sigma_{v}^{2}}
\end{aligned}
$$

Proof : See appendix A.1.

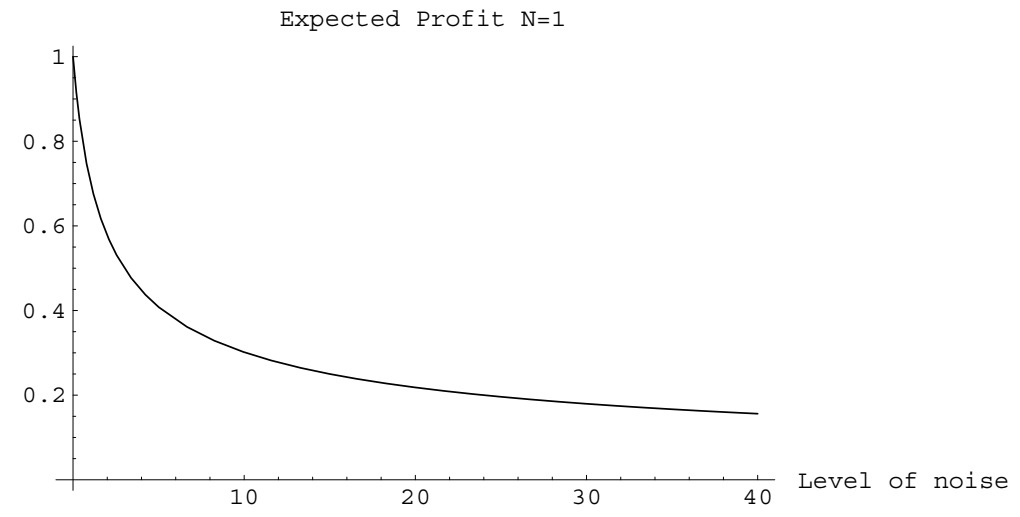

Figure 3: Expected Profit $(\mathrm{N}=1)$.

Figure 3 represents the expected profit achieved by the monopoly as a function of the noise and where the normalization is taken with respect to the perfectly informed case. We observe that the expected profit is a decreasing function of the noise and at the limit is equal to zero. This illustrates that in this linear setting a monopoly is always worse-off collecting a noisier information. As a consequence, it is never optimal ex-ante to add noise to his own information. We now turn to the oligopoly results. 
Proposition 3.3 : There exists a unique linear equilibrium defined by $i=1, \ldots, N, \widetilde{x}_{i}=$ $\beta_{i}^{*}(\sigma) \widetilde{S}_{i}$ and $\widetilde{p}=\lambda^{*}(\sigma) \widetilde{\omega}$ and where $\left(\beta_{1}^{*}, \ldots, \beta_{N}^{*}, \lambda^{*}\right)(\sigma)$ is given by:

$$
\begin{aligned}
& \beta_{i}^{*}(\sigma)=\frac{\sigma_{u}}{\sigma_{v}} \frac{1}{\left\{\sum_{j=1}^{N} \frac{1+\tau_{j}}{\left(1+2 \tau_{j}\right)^{2}}\right\}^{\frac{1}{2}} \frac{1}{1+2 \tau_{i}}, \quad i=1, \ldots, N,} \\
& \frac{1}{\lambda^{*}(\sigma)}=\frac{\sigma_{u}}{\sigma_{v}} \frac{1}{\left\{\sum_{j=1}^{N} \frac{1+\tau_{j}}{\left(1+2 \tau_{j}\right)^{2}}\right\}^{\frac{1}{2}}}\left[1+\sum_{j=1}^{N} \frac{1}{1+2 \tau_{j}}\right], \text { with } \tau_{j}=\frac{\sigma_{j}^{2}}{\sigma_{v}^{2}}, j=1 \ldots, N .
\end{aligned}
$$

Proof : See appendix A.2.

To the best of our knowledge, this result generalizes all the existing ones in the literature. More precisely, Kyle (1984), Admati and Pfleiderer (1988b) show the existence of a linear equilibrium while assuming that, i) the pricing rule is linear, ii) each informed agent conjectures that each other informed trader's strategy is linear in his signal (and consequently that at equilibrium informed traders strategies are linear). This does not prove in any case, i) the uniqueness of the linear equilibrium ${ }^{8}$, ii) that the class of possible linear equilibria corresponds to linear reaction of each informed agent in their signal.

In light of this, proposition 3.3 states that, the class of linear equilibria is indeed reduced to a singleton and that the reaction of each informed agent is, at the equilibrium, linear.

In the next proposition we determine the expected profits of the traders at the individual and aggregated level.

\section{Proposition 3.4 :}

- The expected profit $\pi_{i}^{*}(\sigma)$ for the agent $i$ is, at equilibrium, given by:

$$
\begin{aligned}
& \pi_{i}^{*}(\sigma)=\frac{\sigma_{v} \sigma_{u} \frac{1+\tau_{i}}{\left(1+2 \tau_{i}\right)^{2}}}{\left\{\sum_{j=1}^{N} \frac{1+\tau_{j}}{\left(1+2 \tau_{j}\right)^{2}}\right\}^{\frac{1}{2}}\left\{1+\sum_{j=1}^{N} \frac{1}{1+2 \tau_{j}}\right\}}, \quad i=1, \ldots, N, \\
& \text { with } \tau_{i}=\frac{\sigma_{i}^{2}}{\sigma_{v}^{2}} .
\end{aligned}
$$

For $\sigma=0$, we obtain the benchmark:

$$
\pi_{i}^{*}(0, N)=\frac{\sigma_{v} \sigma_{u}}{(N+1) \sqrt{N}} .
$$

\footnotetext{
${ }^{8} \mathrm{We}$ emphasize here that linear equilibrium refers to the linearity in price according to definition 2.2 and not to linearity in informed traders strategies.
} 
- The expected aggregate profit $\pi^{*}(\sigma)$ is, at equilibrium, given by:

$$
\begin{aligned}
& \pi^{*}(\sigma)=\sum_{i=1}^{N} \pi_{i}^{*}(\sigma)=\frac{\sigma_{v} \sigma_{u}\left\{\sum_{j=1}^{N} \frac{1+\tau_{j}}{\left(1+2 \tau_{j}\right)^{2}}\right\}^{\frac{1}{2}}}{1+\sum_{j=1}^{N} \frac{1}{1+2 \tau_{j}}}, \\
& \text { with } \tau_{j}=\frac{\sigma_{j}^{2}}{\sigma_{v}^{2}}, \quad j=1, \ldots, N . \\
& \text { For } \sigma=0 \text {, we have } \pi^{*}(0, N)=\frac{\sigma_{v} \sigma_{u} \sqrt{N}}{N+1} .
\end{aligned}
$$

Proof : See appendix A.2. 


\section{Equilibrium Properties}

Proposition 4.1 : At equilibrium, the individual reaction $\beta_{i}^{*}(\sigma)$ of the agent $i$ is decreasing with each other competitor's available information; that is to say, increasing with $\sigma_{j}, j \neq i$, and increasing with its own information precision, that is to say, decreasing with $\sigma_{i}$.

This proposition shows that the informed traders react all the more to their private information than the other traders' information is noisy. Indeed, since the total amount of noise in the market increases the traders can camouflage more their information.

Proof : See appendix B.1.

Proposition 4.2 : For $i=1, \ldots, N$, we define:

$$
\begin{aligned}
& b\left(\tau_{-i}\right)=1-\sum_{j \neq i} \frac{3+2 \tau_{j}}{\left(1+2 \tau_{j}\right)^{2}}, \\
& c\left(\tau_{-i}\right)=2+\sum_{j \neq i} \frac{2 \tau_{j}-1}{\left(1+2 \tau_{j}\right)^{2}},
\end{aligned}
$$

then:

- if $b\left(\tau_{-i}\right) \geq 0$, the liquidity $\lambda^{*^{-1}}(\tau)$ (or $\left.\lambda^{*^{-1}}(\sigma)\right)$ is, at equilibrium, an increasing function of $\tau_{i}=\frac{\sigma_{i}^{2}}{\sigma_{v}^{2}}$ for any fixed $\tau_{-i}$,

- if $b\left(\tau_{-i}\right) \leq 0$ and $c\left(\tau_{-i}\right) \leq 0$, the liquidity $\lambda^{*^{-1}}(\tau)$ (or $\lambda^{*^{-1}}(\sigma)$ ) is, at equilibrium, a decreasing function of $\tau_{i}=\frac{\sigma_{i}^{2}}{\sigma_{v}^{2}}$ for any fixed $\tau_{-i}$,

- if $b\left(\tau_{-i}\right) \leq 0$ and $c\left(\tau_{-i}\right) \geq 0$, then:

* for $\tau_{i} \leq-\frac{c\left(\tau_{-i}\right)}{2 b\left(\tau_{-i}\right)}$, the liquidity $\lambda^{*^{-1}}(\tau)$ (or $\left.\lambda^{*^{-1}}(\sigma)\right)$ is, at equilibrium, an increasing function of $\tau_{i}=\frac{\sigma_{i}^{2}}{\sigma_{v}^{2}}$ for any fixed $\tau_{-i}$,

* for $\tau_{i} \geq-\frac{c\left(\tau_{-i}\right)}{2 b\left(\tau_{-i}\right)}$, the liquidity $\lambda^{*^{-1}}(\tau)$ (or $\left.\lambda^{*^{-1}}(\sigma)\right)$ is, at equilibrium, a decreasing function of $\tau_{i}=\frac{\sigma_{i}^{2}}{\sigma_{v}^{2}}$ for any fixed $\tau_{-i}$,

Remark 4.1 : All the previous cases are possible.

Proof : See appendix B.2.

\section{Proposition 4.3 :}


- For $i=1, \ldots, N$, the individual profit $\pi_{i}^{*}(\sigma)$ is, at equilibrium, an increasing function of $\sigma_{-i}$ the $(N-1)$ vectorial noise corresponding to each other informed competitor's noise and a decreasing function of one's own noise $\sigma_{i}$.

- $\quad$ * If $b\left(\tau_{-i}\right) \geq 0$, the aggregate profit $\pi^{*}(\sigma)$ is, at equilibrium, a decreasing function of the noise $\tau_{i}=\frac{\sigma_{i}^{2}}{\sigma_{v}^{2}}$ for $i=1, \ldots, N$ and any fixed $\tau_{-i}$.

* If $b\left(\tau_{-i}\right) \leq 0$ and $c\left(\tau_{-i}\right) \leq 0$, the aggregate profit $\pi^{*}(\sigma)$ is, at equilibrium, an increasing function of the noise $\tau_{i}=\frac{\sigma_{i}^{2}}{\sigma_{v}^{2}}$ for $i=1, \ldots, N$ and any fixed $\tau_{-i}$.

* If $b\left(\tau_{-i}\right) \leq 0$ and $c\left(\tau_{-i}\right) \geq 0$ then:

- For $\tau_{i} \leq-\frac{c\left(\tau_{-i}\right)}{2 b\left(\tau_{-i}\right)}$, the aggregate profit $\pi^{*}(\sigma)$ is, at equilibrium, a decreasing function of the noise $\tau_{i}=\frac{\sigma_{i}^{2}}{\sigma_{v}^{2}}$ for $i=1, \ldots, N$ and any fixed $\tau_{-i}$.

- For $\tau_{i} \geq-\frac{c\left(\tau_{-i}\right)}{2 b\left(\tau_{-i}\right)}$, the aggregate profit $\pi^{*}(\sigma)$ is, at equilibrium, an increasing function of the noise $\tau_{i}=\frac{\sigma_{i}^{2}}{\sigma_{v}^{2}}$ for $i=1, \ldots, N$ and any fixed $\tau_{-i}$.

Remark 4.2 : As already pointed out, each case can be envisioned.

Proof : See appendix B.3.

Remark 4.3 : Proposition 4.3 exactly illustrates the trade-off competition versus "noise" behavior.

The interpretations ${ }^{9}$ of propositions $4.2-4.3$ are as follows. First as in Kyle (1985), we still have that the depth $\left(\lambda^{-1}\right)$ and the aggregate expected profit are inversely related. Second, in our framework, the liquidity in the market and the aggregate expected profit are not monotonic functions of the set of information in the market. In particular,

- if there is a sufficient amount of noise in the market $b\left(\tau_{-i}\right) \geq 0$, then the more precise is the collected information by an insider the bigger is the depth and the smaller is the aggregate expected profit. In other words, both price pressure and aggregate expected profit increase with the precision of the information collected by one trader all things being equal.

- if the amount of noise in the market is small enough (but not too small)- $b\left(\tau_{-i}\right) \leq 0$ and $c\left(\tau_{-i}\right) \geq 0$ - as long as the collected information is relevant - $\left(\tau_{i} \leq-\frac{c\left(\tau_{-i}\right)}{2 b\left(\tau_{-i}\right)}\right)$ - the price pressure and the aggregate expected profit increase with the precision of the information collected by one trader all things being equal. If not $-\tau_{i} \geq-\frac{c\left(\tau_{-i}\right)}{2 b\left(\tau_{-i}\right)}$ - the price pressure and the aggregate expected profit decrease with the precision of the information collected by one trader all things being equal.

\footnotetext{
${ }^{9}$ It should be mentioned that the aggregate profit is proportional to $\lambda$ by a factor $\sigma_{u}^{2}$. Therefore the properties for the aggregate profits can be deduced from those of the liquidity.
} 
- For small levels of noise $-b\left(\tau_{-i}\right) \leq 0$ and $c\left(\tau_{-i}\right) \leq 0$ - the price pressure and the aggregate expected profit decrease with the precision of the information collected by one trader all things being equal.

To summarize the previous effects, a little amount of noise in the market does not allow for price manipulation. Indeed, in the latter case, the cost of noise supersedes the price manipulation since the market are in some sense still nearly efficient (take the polar case where a large number of nearly perfectly informed traders compete with a single less informed trader). On the contrary a larger amount of noise in the market enables price manipulation. This effect is bigger than the cost of noise. Finally, for intermediate amount of noise in the market, we observe different regions for which one effect dominates the other one.

Proposition 4.4 : Let $N$ informed agents endowed with signals $\widetilde{S}_{i}=\widetilde{v}+\widetilde{\varepsilon}_{i}$ with $\left\{\widetilde{\varepsilon}_{1}, \ldots, \widetilde{\varepsilon}_{N}, \widetilde{v}, \widetilde{u}\right\}$ mutually independent and where $\widetilde{u}$ corresponds to the noise trading, $\widetilde{v}$ the liquidation value of the risky asset and $\widetilde{x}_{i}=\beta_{i}^{*}(\sigma) \widetilde{S}_{i}$ the orders submitted by each competing informed traders at equilibrium, then if we define $\Sigma_{N}$ as:

$$
\begin{aligned}
& \Sigma_{N}=\left\{\sigma=\left(\sigma_{1}, \ldots, \sigma_{N}\right)^{\prime} \in \mathbb{R}_{+}^{N}\right\} \\
& \forall \sigma \in \Sigma_{N}, \forall i=1, \ldots, N \\
& \pi_{i}^{*}\left(\sigma_{1}, \ldots, \sigma_{N}\right) \geq \pi_{i}^{*}(0, \ldots, 0)=\pi_{i}^{*}(0, N), \\
& \exists i_{\circ} \in\{1, \ldots, N\} / \pi_{i_{\circ}}^{*}\left(\sigma_{1}, \ldots, \sigma_{N}\right)>\pi_{i_{\circ}}^{*}(0, \ldots, 0) .
\end{aligned}
$$

We have:

- for $N \geq 4 \Sigma_{N}$ is non-empty. In other words, noise is ex-ante optimal.

- For $N \leq 3, \Sigma_{N}$ is empty.

Proof : See appendix B.4.

The interpretation of the proposition is the following. The presence of noise mitigates the competition. 


\section{Symmetric Case}

We focus in this section on the symmetric heterogeneous beliefs case that is when all informed agents are endowed with signals of same precision, i.e., $\sigma_{i}=\sigma$ for $i=1, \ldots, N$.

We still maintain the mutual independence of $\left\{\widetilde{\varepsilon}_{1}, \ldots, \widetilde{\varepsilon}_{N}, \widetilde{v}, \widetilde{u}\right\}$. Then we can state as corollaries of the previous propositions $3.3-4.3$ that:

Proposition 5.1 : There exists a unique perfect Bayesian linear equilibrium defined as in proposition 3.3. In this particular case $\left(\beta_{1}^{*}, \ldots, \beta_{N}^{*}, \lambda^{*}\right)(\sigma, N)$ is characterized by:

$$
\begin{aligned}
& \beta_{i}^{*}(\sigma, N)=\beta^{*}(\sigma, N)=\frac{\sigma_{u}}{\sigma_{v}} \frac{1}{\sqrt{N} \sqrt{1+\frac{\sigma^{2}}{\sigma_{v}^{2}}}}, \quad i=1, \ldots, N, \\
& \frac{1}{\lambda^{*}(\sigma, N)}=\frac{\sigma_{u}}{\sigma_{v}} \frac{N+1+2 \frac{\sigma^{2}}{\sigma_{v}^{2}}}{\sqrt{N} \sqrt{1+\frac{\sigma^{2}}{\sigma_{v}^{2}}}}
\end{aligned}
$$

The individual profit $\pi_{i}^{*}(\sigma, N)$, for $i=1, \ldots, N$ is, at equilibrium, given by:

$$
\pi_{i}^{*}(\sigma, N)=\sigma_{v} \sigma_{u} \frac{\sqrt{1+\frac{\sigma^{2}}{\sigma_{v}^{2}}}}{\sqrt{N}\left(N+1+\frac{2 \sigma^{2}}{\sigma_{v}^{2}}\right)},
$$

and the aggregate profit $\pi^{*}(\sigma, N)$ is, at equilibrium, given by:

$$
\pi^{*}(\sigma, N)=\sigma_{v} \sigma_{u} \frac{\sqrt{N} \sqrt{1+\frac{\sigma^{2}}{\sigma_{v}^{2}}}}{N+1+\frac{2 \sigma^{2}}{\sigma_{v}^{2}}} .
$$

Proof : the results correspond to the applications of the previous propositions to the symmetric case.

Figure 4 represents, in the symmetric case, the reaction of each informed agent $\beta_{i}^{*}(\sigma, N)$ as a function of the number of insiders and the level of noise, which is measured by $\frac{\sigma^{2}}{\sigma_{v}^{2}} \cdot{ }^{10}$ We can see that:

- for each given level of noise, the individual reaction function is as usual decreasing with the number of insiders,

\footnotetext{
${ }^{10}$ In the whole paper and in order to draw the graphs, we have set as a normalization $\sigma_{v}=\sigma_{u}=1$.
} 


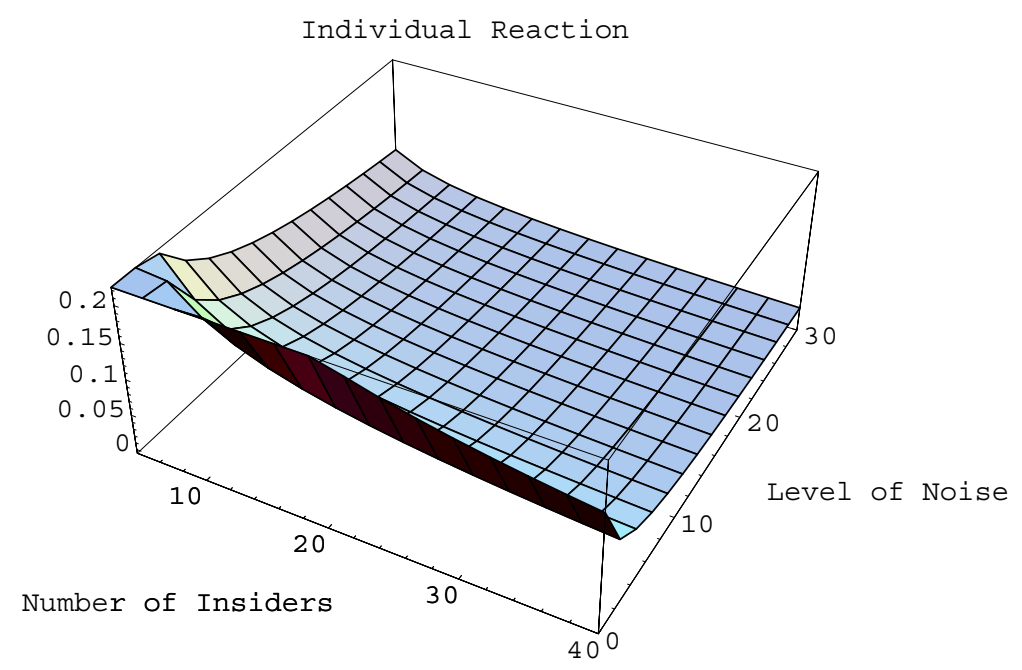

Figure 4: Individual Reaction.

- for each given number of insiders, the individual reaction function is decreasing with the level of noise.

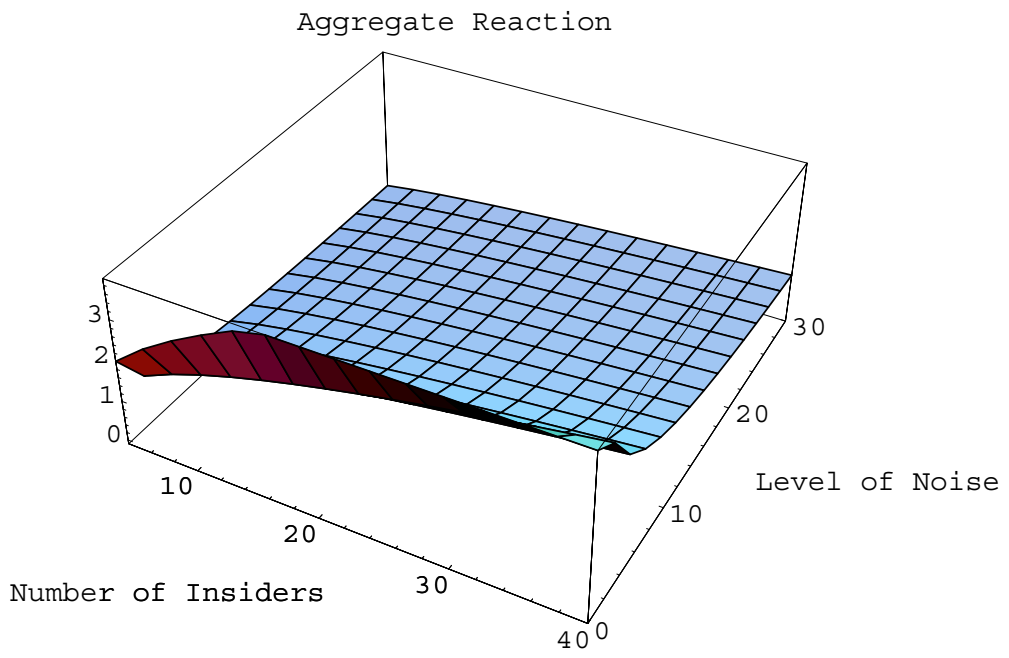

Figure 5: Aggregate Reaction.

Figure 5 represents, in the symmetric case, a kind of aggregate reaction $N \beta^{*}(\sigma, N)$ as a function of the number of insiders and the level of noise. We can notice that:

- for each given level of noise, the aggregate reaction is increasing with the number of insiders,

- for each given number of informed traders, the aggregate reaction is decreasing with the level of noise. 


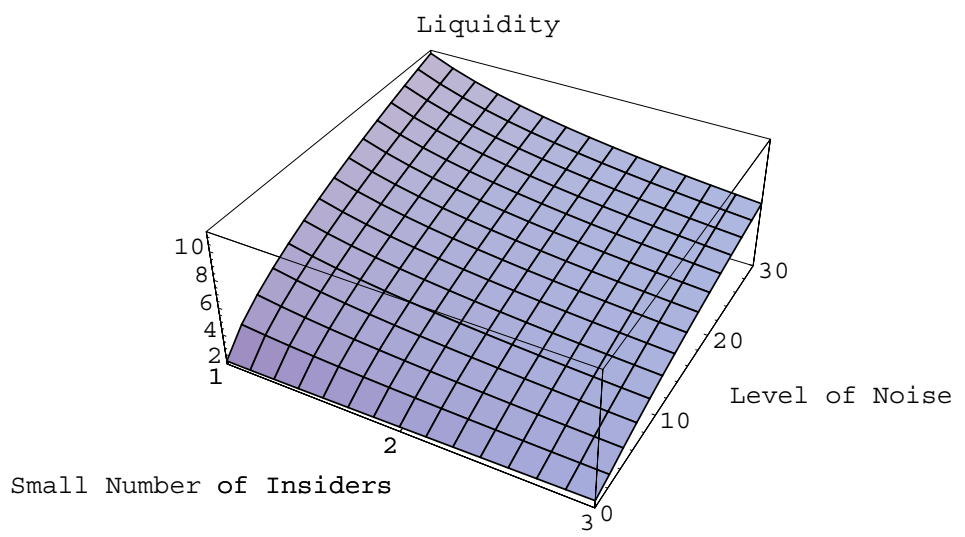

Figure 6: Liquidity $\mathbf{N}<\mathbf{4}$.

Figures 6 and 7 represent, in the symmetric case, the liquidity $\frac{1}{\lambda^{*}}(\sigma, N)$ as a function of both the number of insiders and the level of noise:

- First, for a fixed positive level of noise $\sigma>0$, the liquidity is first decreasing with the number of insiders to reach a minimum at the value $N=1+2{\frac{\sigma^{2}}{\sigma_{v}^{2}}}^{11}$ along the given hyperplan and then increasing with the number of insiders. This seriously differs from the case of perfect information $\sigma=0$ where the liquidity is always increasing with the number of informed traders.

- Second, for a small number of insiders $(N \leq 3)$, we observe as usual, that the liquidity is an increasing function of the level of noise for a fixed number of informed traders.

- Third, for a larger number of informed traders $(N \geq 4)$, and any given number of insiders, the liquidity is first decreasing with the level of noise to reach a minimum at the value $\sigma=\sigma^{*}(N)=\sqrt{\frac{N-3}{2}} \sigma_{v}$ along the given hyperplan and then increasing with the level of noise. In this case, we observe a "basin".

Figures 8 and 9 represent, in the symmetric case, the individual profit achieved by each informed agent as a function of both the level of noise and the number of insiders. For any fixed level of noise, the individual profit is always decreasing with the number of insiders.

\footnotetext{
${ }^{11}$ Of course, this implicitly assumes that we are extending the variables $N$ to the real axis. For sake of clarity, we will not discuss here in further details the fact that in practice $N \in \mathbb{N}$ the set of positive integers. Indeed one has rather to take the integer part and to discuss whether it is greater than one. See however proposition 5.3 for the exact statements.
} 


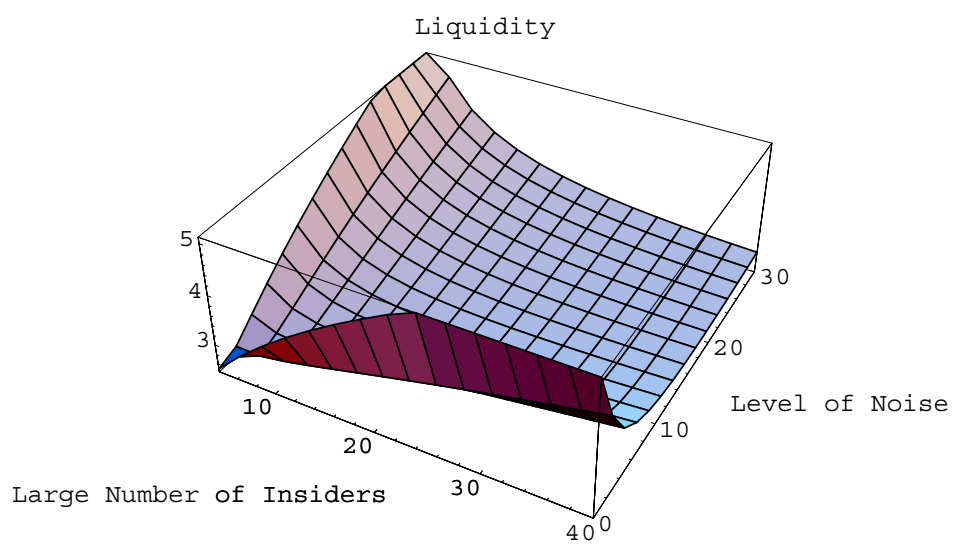

Figure 7: Liquidity $\mathbf{N} \geq \mathbf{4}$.

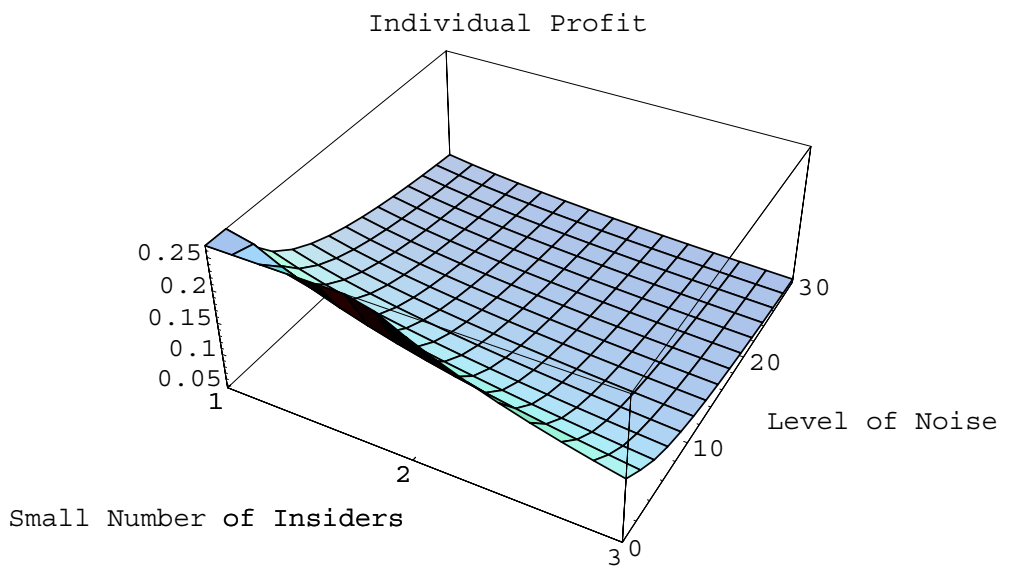

Figure 8: Individual Profit $\mathbf{N}<\boldsymbol{4}$. 
- For a small number of informed agents $(N \leq 3)$, the individual profit is always decreasing with the level of noise for any fixed number of insiders.

- For a large number of informed traders $(N \geq 4)$, and any given number $N$ of insiders, the individual profit is first increasing with the noise to reach a maximum at the value $\sigma=\sigma^{*}(N)=\sqrt{\frac{N-3}{2}} \sigma_{v}$ and then decreasing with the level of noise.

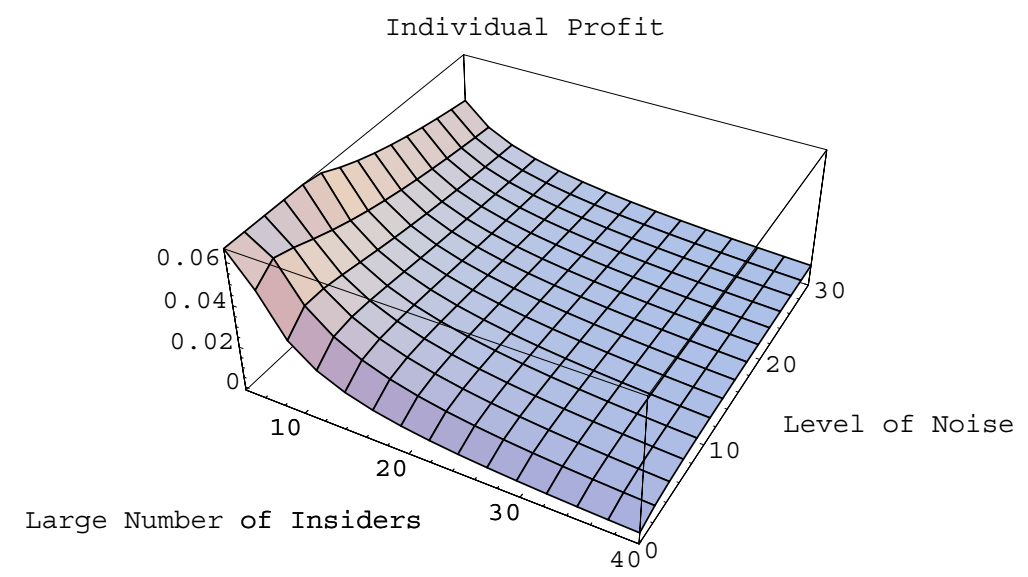

Figure 9: Individual Profit $\mathbf{N} \geq \mathbf{4}$.

This illustrates the idea that noise has basically two effects, one costly which is due to the fact that, in some sense and for a part of his trade, the insider is behaving as a noise trader; and the other beneficial since it tends to weaken the competition. In other words, for a large number of insiders the beneficial effect of noise supersedes the costly one.

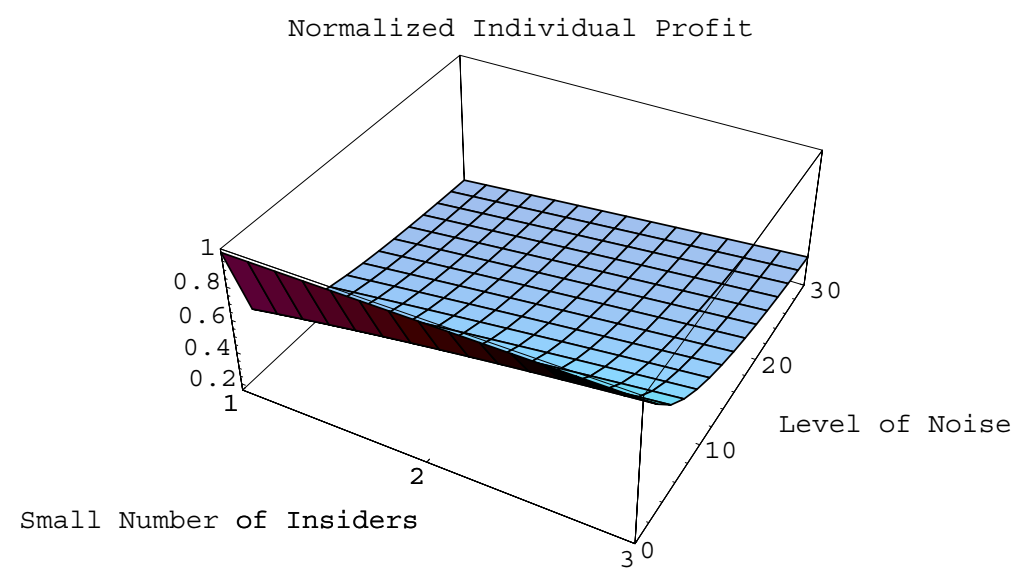

Figure 10: Normalized Individual Profit $\mathbf{N}<\mathbf{4}$. 
Figures 10 and 11 represent, in the symmetric case, the normalized individual profit achieved by each informed agent as a function of both the level of noise and the number of insiders. We have chosen the normalization with respect to the profit $\pi_{i}^{*}(0, N)$ performed in case of perfect information $\sigma=0$. In other words, we have reported the ratio $\frac{\pi_{i}^{*}(\sigma, N)}{\pi_{i}^{*}(0, N)}$ as a function of the number of insiders and the level of noise. Again, two cases have to be envisioned.

- First, for a small number of insiders $(N \leq 3)$, this ratio is decreasing with the level of noise and increasing with the number of informed traders. However for a small number of insiders, this ratio is always smaller than one.

- Second, for a larger number of informed traders $(N \geq 4)$, there exist for each given number of insiders, levels of noise for which this ratio is bigger than one. Moreover, it is increasing with the number of informed traders, for a fixed level of noise. For a given number of insiders, the ratio is first increasing to reach a maximum at the value $\sigma=\sigma^{*}(N)=\sqrt{\frac{N-3}{2}} \sigma_{v}$ in the given hyperplan $(N)$ and then decreasing with the level of noise.

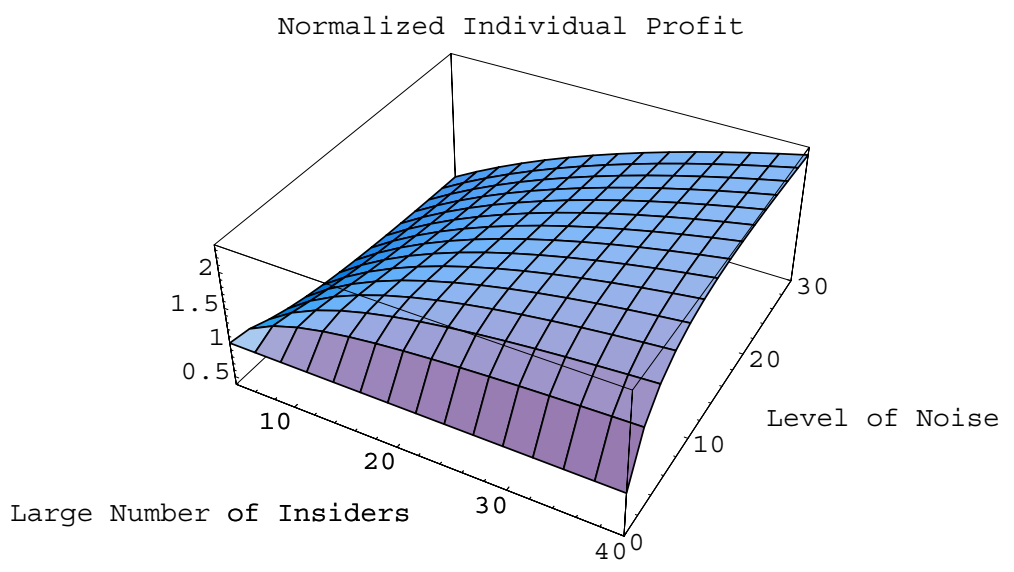

Figure 11: Normalized Individual Profit $\mathbf{N} \geq \mathbf{4}$.

Figures 12 and 13 represent the normalized aggregate profit as a function of the level of noise and the number of insiders. The normalization is performed with respect to the profit achieved by the monopoly in Kyle (1985) $\operatorname{setup}\left(\pi^{*}(0,1)=\frac{1}{2} \sigma_{u} \sigma_{v}\right)$.

- For any fixed positive level of noise $\sigma>0$, the aggregate profit is first increasing with the 


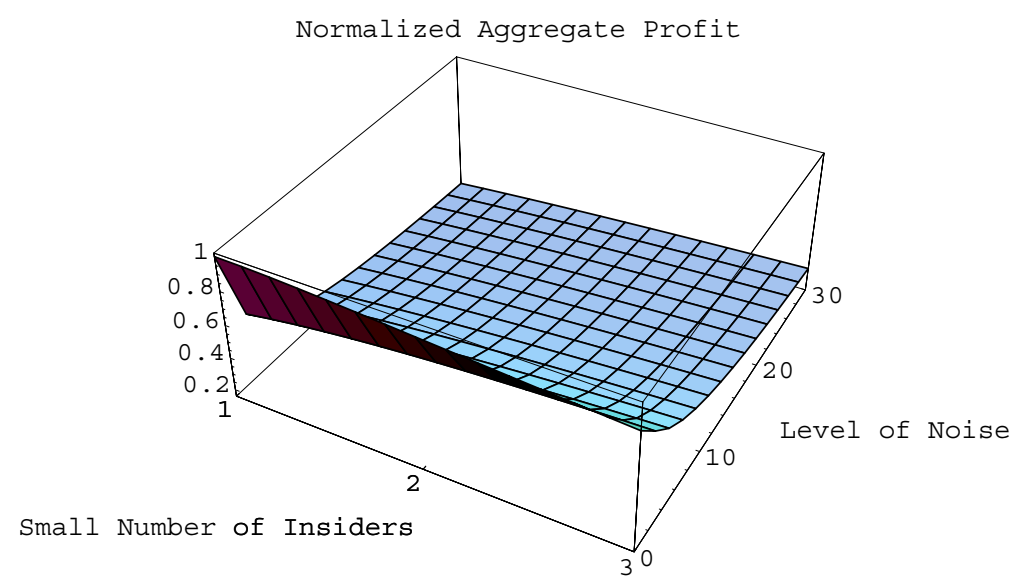

Figure 12: Normalized Aggregate Profit $\mathbf{N}<\mathbf{4}$.

number of insiders to reach a maximum in the given hyperplan at the value $N=1+2{\frac{\sigma^{2}}{\sigma_{v}^{2}}}^{12}$. Again, this result has to be compared with the perfect information case where the aggregate profit is always decreasing with the number of insiders.

- For a small and fixed number of informed traders $(N \leq 3)$, the aggregate profit is always decreasing with the level of noise.

- For a larger and given number of informed traders $(N \geq 4)$, the aggregate profit is first increasing with the level of noise to reach a maximum at the value $\sigma=\sigma^{*}(N)=\sqrt{\frac{N-3}{2}} \sigma_{v}$ and then decreasing with the level of noise. Again, this has to be related to the two effects (beneficial and costly ones) of the noise.

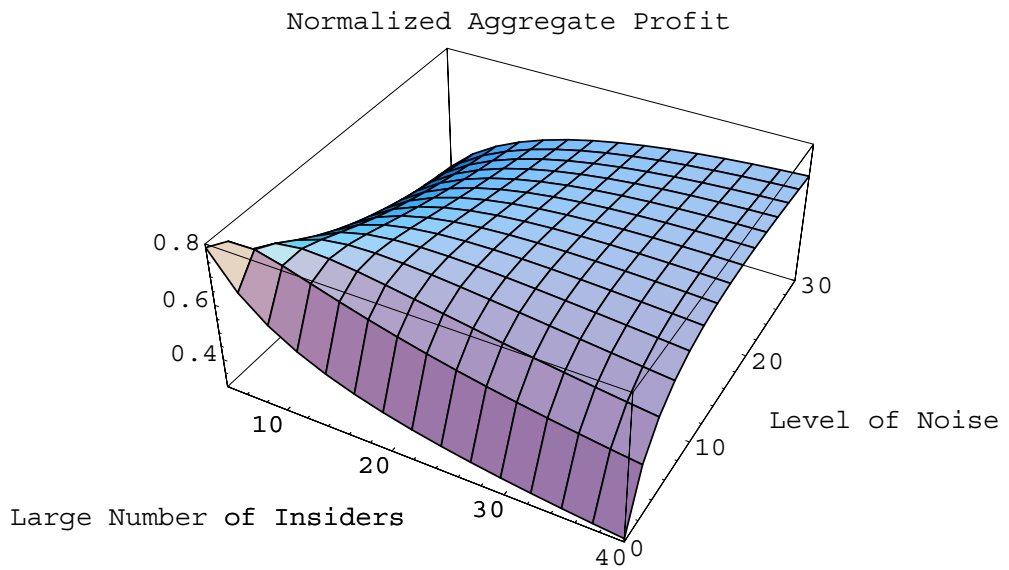

Figure 13: Normalized Aggregate Profit $\mathbf{N} \geq \mathbf{4}$.

\footnotetext{
${ }^{12}$ The same remarks as for the liquidity apply, namely, the fact that $1+2 \frac{\sigma^{2}}{\sigma_{v}^{2}}$ is not necessarily an integer. See however proposition 5.3 for the exact statements.
} 
Proposition 5.2 : For all $N \geq 4$, and for all $\sigma \in\left(0, \frac{\sigma_{v} \sqrt{N+1} \sqrt{N-3}}{2}\right)$, we have:

$$
\forall i=1, \ldots, N, \quad \pi_{i}^{*}(\sigma, N)>\pi_{i}^{*}(0, N)
$$

In other words, noise is preferable. And furthermore we have an equivalence result:

$$
\pi_{i}^{*}(\sigma, N)>\pi_{i}^{*}(0, N) \Longleftrightarrow \sigma \in\left(0, \frac{\sigma_{v} \sqrt{N+1} \sqrt{N-3}}{2}\right) .
$$

Proof : See appendix C.1.

Proposition 5.2 delineates the set of possible noise that are preferable for each informed traders.

Proposition 5.3 : If we define $N^{*}(\sigma)$ as:

$$
\begin{aligned}
& N^{*}(\sigma)=1+2 \frac{\sigma^{2}}{\sigma_{v}^{2}}, \text { if } 1+2 \frac{\sigma^{2}}{\sigma_{v}^{2}} \in \mathbb{I N}, \\
& N^{*}(\sigma)=\underset{N \in\left(\widetilde{N}_{1}(\sigma), \widetilde{N}_{2}(\sigma)\right)}{\operatorname{argmax}} \pi^{*}(N, \sigma), \text { if } 1+2 \frac{\sigma^{2}}{\sigma_{v}^{2}} \notin \mathbb{N}, \\
& \text { and } \tilde{N}_{1}(\sigma)=\operatorname{int}\left(1+2 \frac{\sigma^{2}}{\sigma_{v}^{2}}\right), \\
& \tilde{N}_{2}(\sigma)=\widetilde{N}_{1}(\sigma)+1,
\end{aligned}
$$

then the aggregate profit $\pi^{*}(N, \sigma)$ is at equilibrium maximized for the value $N=N^{*}(\sigma)$.

Proof : For each given level of noise $\sigma$, the aggregate profit $\pi^{*}(\sigma, N)$ is maximized for the value $\tilde{N}(\sigma)=1+2 \frac{\sigma^{2}}{\sigma_{v}^{2}}$. Therefore if taking into account the possible outcome for $\tilde{N}(\sigma)$ as an integer or not, the result is obtained.

Proposition 5.3 exactly states that for a given level of available precision $\sigma$, there exists an optimal size of the market $N^{*}(\sigma)$, for which the aggregate profit is maximized.

Proposition 5.4 : For each level of precision and number of informed traders $(\sigma, N)$, the informativeness of prices $I(\sigma, N)$ is given at equilibrium by:

$$
I(\sigma, N)=\{\operatorname{Var}(\widetilde{v} / \widetilde{p})\}^{-1}=\{\operatorname{Var}(\widetilde{v} / \widetilde{w})\}^{-1}=\frac{1}{\sigma_{v}^{2}} \frac{N+1+2 \frac{\sigma^{2}}{\sigma_{v}^{2}}}{1+2 \frac{\sigma^{2}}{\sigma_{v}^{2}}},
$$

Proof : See appendix C.2. 


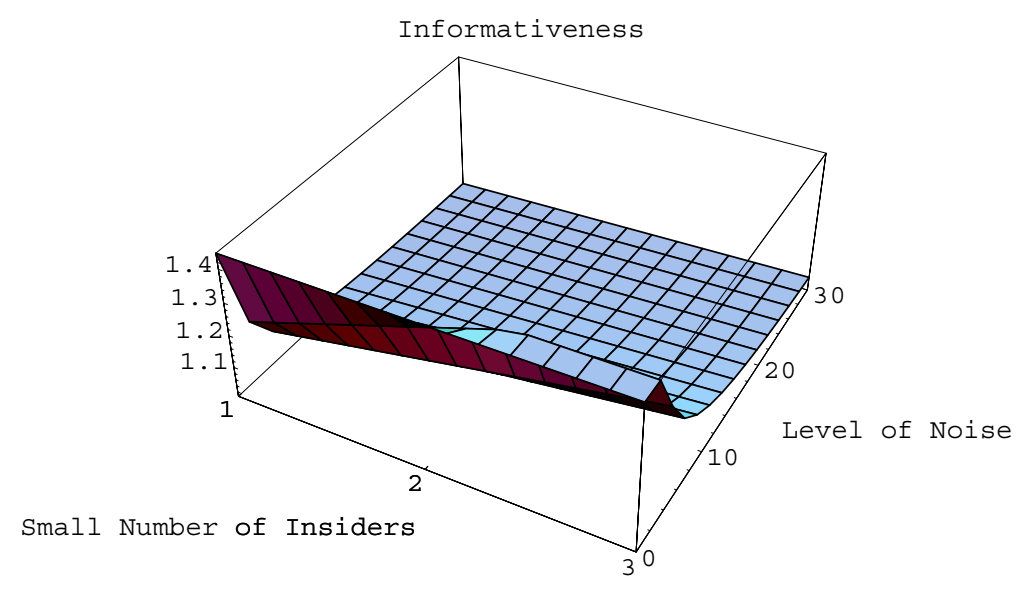

Figure 14: Informativeness $\mathbf{N}<\mathbf{4}$.

Figures 14 and 15 represent the informativeness of prices as a function of both the level of noise and the number of insiders.

- For any given number of insiders, it is always decreasing with the level of noise.

- For any fixed level of noise, the informativeness is always increasing with the number of informed agents.

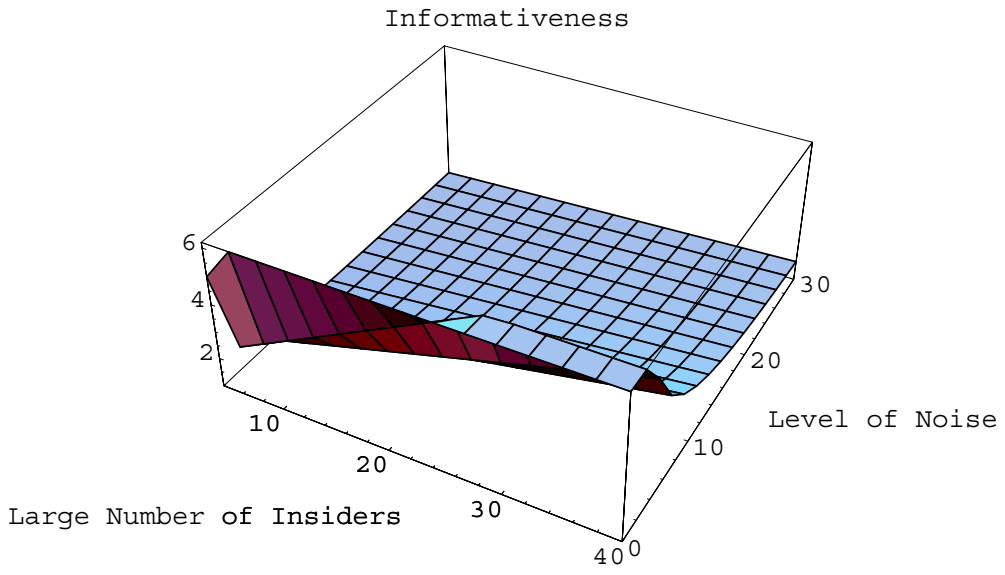

Figure 15: Informativeness $\mathbf{N} \geq \mathbf{4}$.

Proposition 5.5 : There exists an optimal level of noise, for $N \geq 4$ :

$$
\sigma^{*}(N)=\sigma_{v} \sqrt{\frac{N-3}{2}},
$$

which maximizes each individual profit in the symmetric case. 
Proof : See appendix C.3.

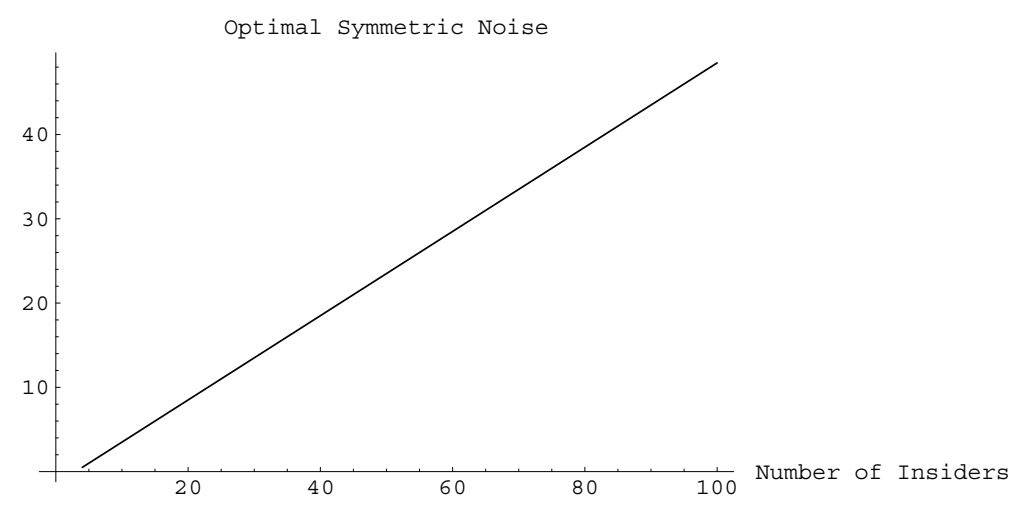

Figure 16: Optimal Level of Noise.

In figure 16, we have plotted the optimal level of noise $\frac{\sigma^{*^{2}}(N)}{\sigma_{v}^{2}}$ as a function of the number of informed agents. It is an affine function of the number of insiders. Therefore, when $N$ is large, the optimal noise is also large.

Proposition 5.6 : For the optimal level of noise $\sigma^{*}(N)=\sigma_{v} \sqrt{\frac{N-3}{2}}$, the individual and aggregate reaction, liquidity, individual profit and aggregate profit are, at equilibrium, given by:

$$
\begin{aligned}
& \beta_{i}^{*}(N)=\frac{\sigma_{u}}{\sigma_{v}} \frac{\sqrt{2}}{\sqrt{N} \sqrt{N-1}}, \\
& N \beta_{i}^{*}(N)=\frac{\sigma_{u}}{\sigma_{v}} \sqrt{\frac{2 N}{N-1}}, \\
& \frac{1}{\lambda^{*}(N)}=\frac{\sigma_{u}}{\sigma_{v}} 2 \sqrt{2} \sqrt{1-\frac{1}{N}}, \\
& \pi_{i}^{*}(N)=\frac{\sigma_{v} \sigma_{u}}{2 \sqrt{2}} \frac{1}{\sqrt{N} \sqrt{N-1}}, \\
& \pi^{*}(N)=\frac{\sigma_{v} \sigma_{u}}{2 \sqrt{2}} \frac{1}{\sqrt{1-\frac{1}{N}}} .
\end{aligned}
$$

Proof : The proofs are just a question of replacement in each of the previous expression and therefore are omitted here. 
These results have to be compared with the symmetric and perfectly informed case where:

$$
\begin{aligned}
& \beta_{i}^{*}(0, N)=\frac{\sigma_{u}}{\sigma_{v}} \frac{1}{\sqrt{N}}, \\
& N \beta_{i}^{*}(0, N)=\frac{\sigma_{u}}{\sigma_{v}} \sqrt{N}, \\
& \frac{1}{\lambda^{*}(0, N)}=\frac{\sigma_{u}}{\sigma_{v}} \frac{N+1}{\sqrt{N}}, \\
& \pi_{i}^{*}(0, N)=\frac{\sigma_{v} \sigma_{u}}{\sqrt{N}(N+1)}, \\
& \pi^{*}(0, N)=\sigma_{v} \sigma_{u} \frac{\sqrt{N}}{N+1} .
\end{aligned}
$$

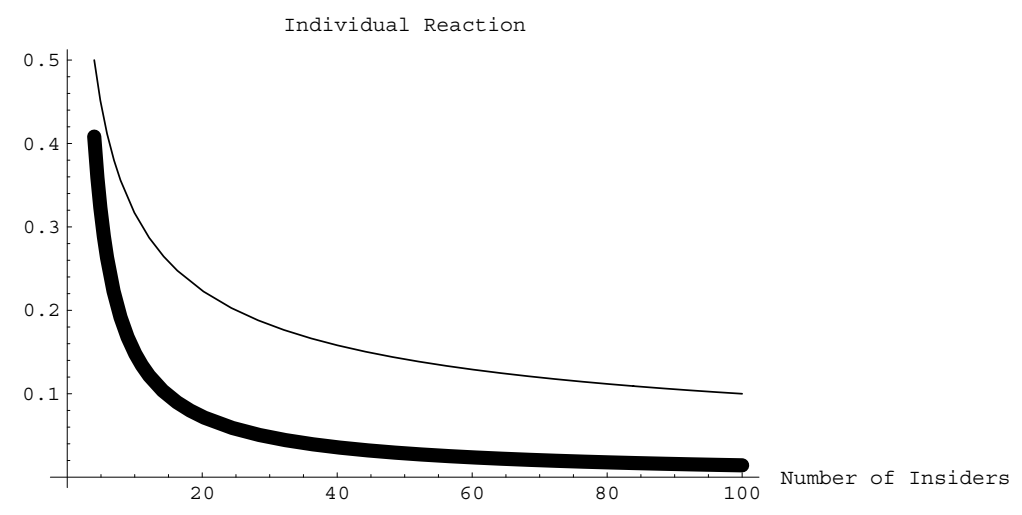

Figure 17: Individual Reaction: Optimal Symmetric Noise versus Perfect Information $\mathbf{N} \geq \mathbf{4}$.

Figure 17 represents the individual reactions $\beta_{i}^{*}(N)$ (thick line) and $\beta_{i}^{*}(0, N)$ (dash line) respectively for the optimal level of noise $\sigma^{*}(N)$ and for the perfect information $\sigma=0$ cases as functions of the number of informed agents. Both reactions are strictly decreasing functions of the number of insiders and converging towards 0 when the number of informed traders is large enough. However the ratio of the two (see figure 18) tends also to 0 when the number of insiders is large enough. This means that the reaction of the insiders in case of optimal noise is "infinitely smaller" than the one observed in the perfect information case when the number of insiders is large. 


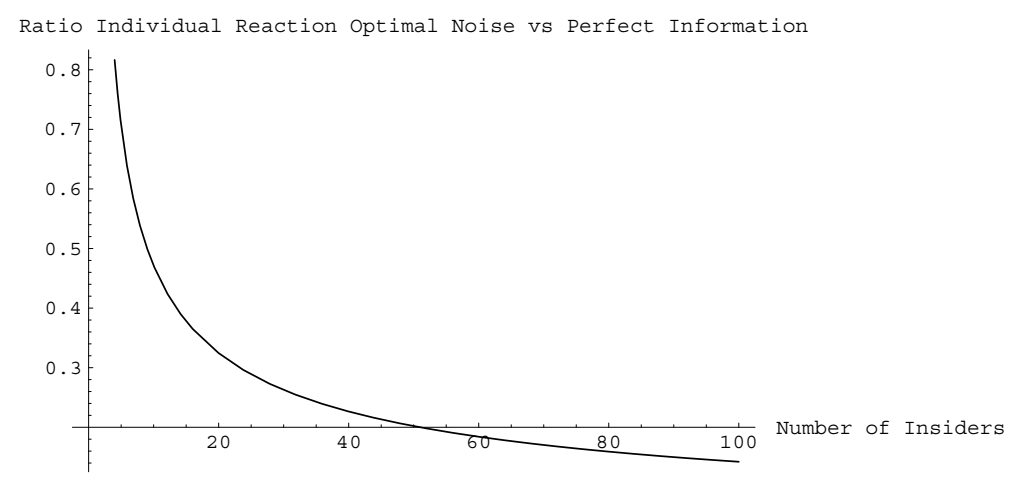

Figure 18: Ratio Individual Reaction: Optimal Symmetric Noise versus Perfect Information $\mathbf{N} \geq 4$.

Figure 19 represents the aggregate reactions $N \beta_{i}^{*}(N)$ (thick line) and $N \beta_{i}^{*}(0, N)$ (dash line) respectively for the optimal level of noise $\sigma^{*}(N)$ and for the perfect information $\sigma=0$ cases as functions of the number of informed agents. In the former case (optimal symmetric noise) the aggregate reaction is a strictly decreasing function of the number of insiders and converging towards a finite positive limit $\frac{\sigma_{u}}{\sigma_{v}} \sqrt{2}$ when the number of informed traders is large enough. In the latter case (perfect information) the aggregate reaction is a strictly increasing function of the number of insiders and converging towards infinity with the number of informed.

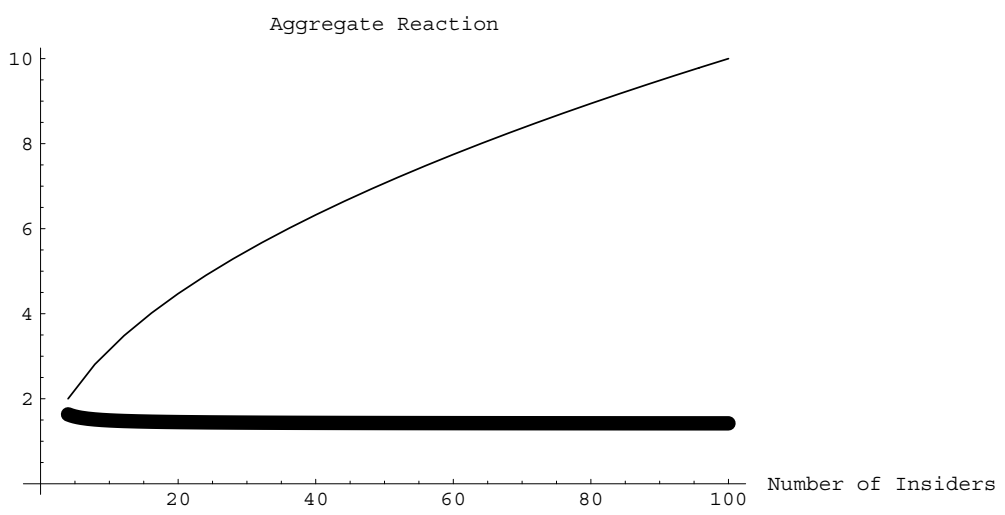

Figure 19: Aggregate Reaction: Optimal Symmetric Noise versus Perfect Information $\mathbf{N} \geq 4$. 


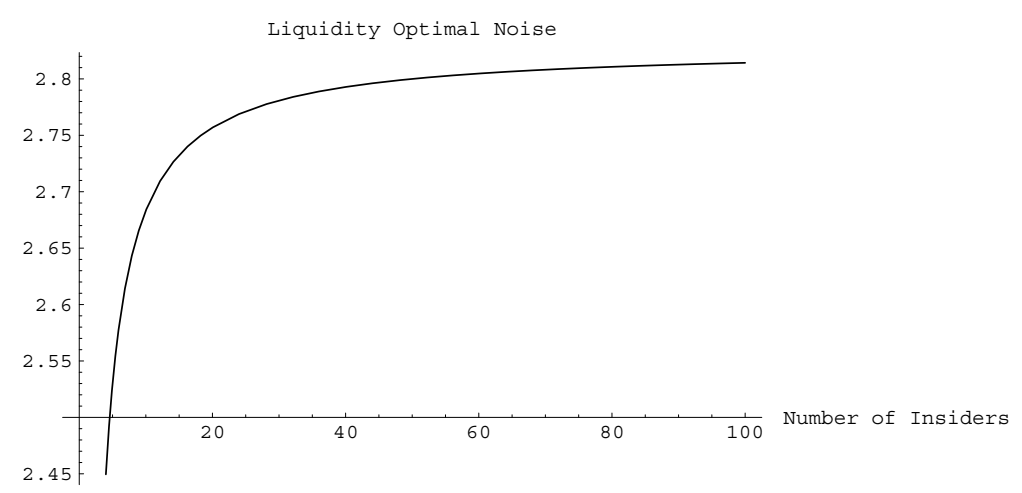

Figure 20: Liquidity Optimal Symmetric Noise $\mathbf{N} \geq \mathbf{4}$.

Figures 20 and 21 represent the liquidity respectively for the optimal level of noise $\sigma=\sigma^{*}(N)$ and the perfect information $\sigma=0$ cases as functions of the number of insiders. In both cases, the liquidity functions are increasing with the number of insiders. However, while in the perfect information case the liquidity tends to infinity with the number of informed agents, in the optimal noise case, it tends to a finite limit $\lambda_{\infty}^{-1}=\frac{\sigma_{u}}{\sigma_{v}} 2 \sqrt{2}$ when the number of insiders is large.

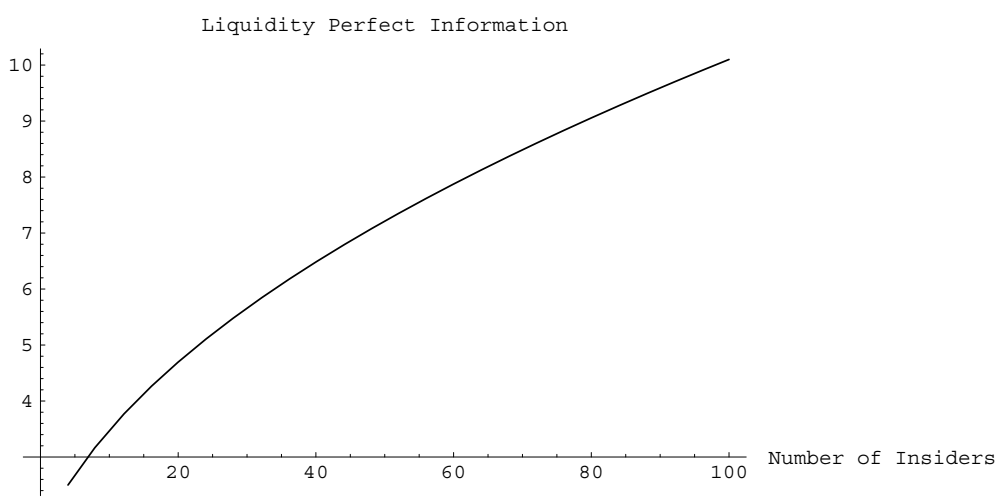

Figure 21: Liquidity Perfect Information $\mathbf{N} \geq \mathbf{4}$.

Figure 22 represents the individual profits $\pi_{i}^{*}(N)$ (thick line) and $\pi_{i}^{*}(0, N)$ (dash line) respectively for the optimal level of noise $\sigma^{*}(N)$ and for the perfect information $\sigma=0$ cases as functions of the number of informed agents. Both individual profits are strictly decreasing functions of the number of insiders and converging towards 0 when the number of informed traders is large enough. However the ratio of the two (see figure 23) tends also to infinity when the number of insiders is large enough. This means that the individual profit achieved by each insider in case 


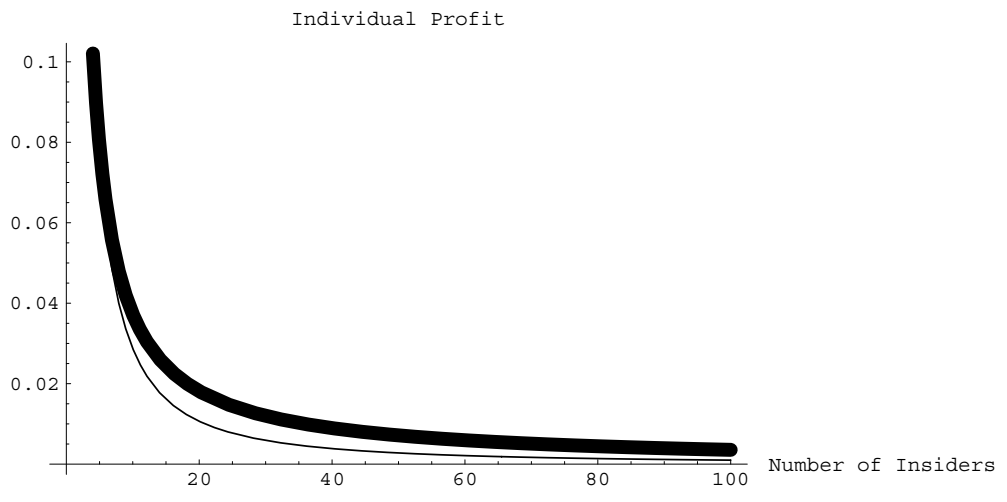

Figure 22: Individual Profit: Optimal Symmetric Noise versus Perfect Information $\mathbf{N} \geq \mathbf{4}$.

of optimal noise is "infinitely greater" than that observed in the perfect information case when the number of insiders is large.

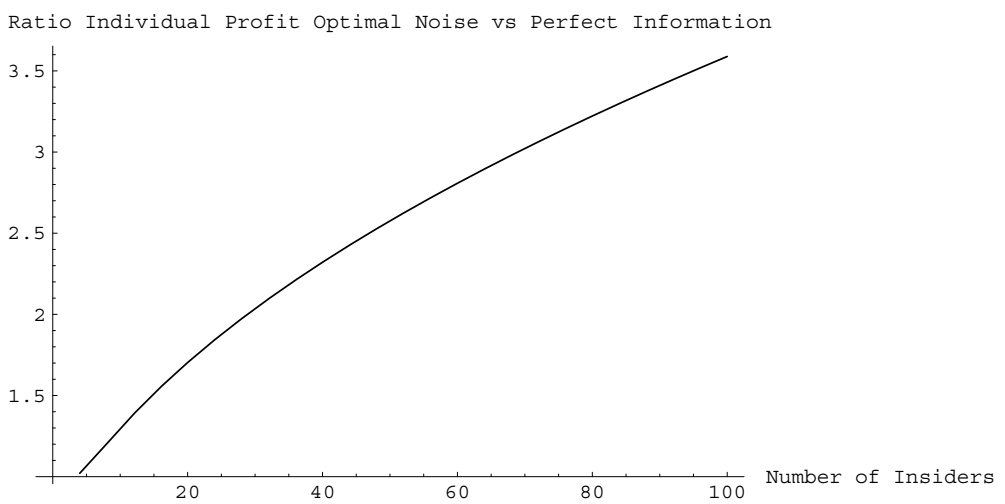

Figure 23: Ratio Individual Profit: Optimal Symmetric Noise versus Perfect Information $\mathbf{N} \geq \mathbf{4}$.

Figure 24 represents the aggregate profits $\pi^{*}(N)$ (thick line) and $\pi^{*}(0, N)$ (dash line) respectively for the optimal level of noise $\sigma^{*}(N)$ and for the perfect information $\sigma=0$ cases as functions of the number of informed agents. Both aggregate profits are strictly decreasing functions of the number of insiders. But while the aggregate profit in the optimal noise case $\sigma^{*}(N)$ has a finite strictly positive limit $\frac{\sigma_{u} \sigma_{v}}{2 \sqrt{2}}$, the aggregate profit converges towards 0 in case of perfect information when the number of informed traders is large enough. 


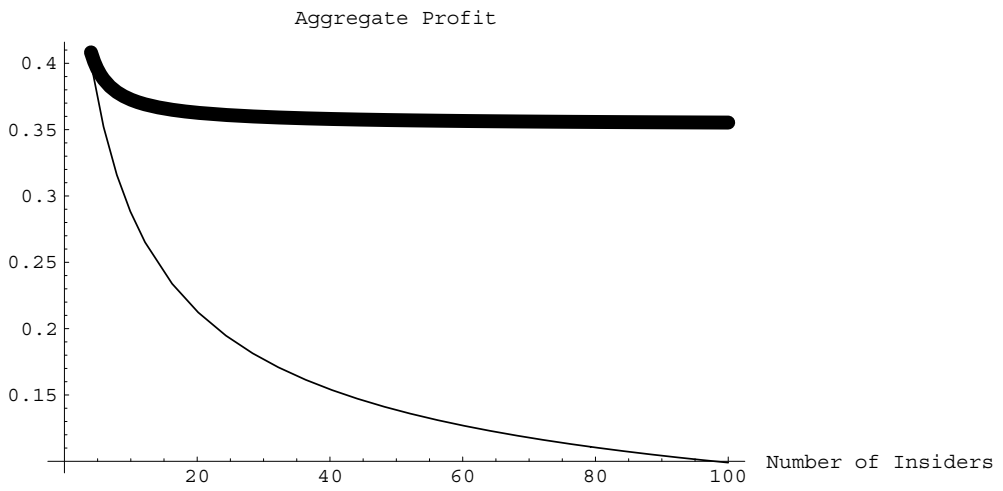

Figure 24: Aggregate Profit: Optimal Symmetric Noise versus Perfect Information $\mathbf{N} \geq \mathbf{4}$.

Proposition 5.7 : When $N$ is large, for the optimal level of noise $\sigma^{*}(N)$ and at equilibrium, we have the following equivalents:

$$
\begin{aligned}
& \beta_{i}^{*}(N) \underset{N \rightarrow+\infty}{\sim} \frac{\sigma_{u}}{\sigma_{v}} \frac{\sqrt{2}}{N}, \\
& N \beta_{i}^{*}(N) \underset{N \rightarrow+\infty}{\sim} \frac{\sigma_{u}}{\sigma_{v}} \sqrt{2}, \\
& \frac{1}{\lambda^{*}(N)} \underset{N \rightarrow+\infty}{\sim} \frac{\sigma_{u}}{\sigma_{v}} 2 \sqrt{2}, \\
& \pi_{i}^{*}(N) \underset{N \rightarrow+\infty}{\sim} \frac{\sigma_{v} \sigma_{u}}{2 \sqrt{2} N}, \\
& \pi^{*}(N) \underset{N \rightarrow+\infty}{\sim} \frac{\sigma_{v} \sigma_{u}}{2 \sqrt{2}} .
\end{aligned}
$$

Proof : The proofs are straightforward and omitted here.

Proposition 5.8 : The informativeness of prices $I^{*}(N)$ is given for the optimal level of noise $\sigma^{*}(N)$ and at equilibrium by:

$$
I^{*}(N)=\operatorname{Var}(\widetilde{v} / w)^{-1}=\frac{1}{\sigma_{v}^{2}} \frac{2(N-1)}{N-2},
$$

and when $N$ is large, $I^{*}(N)$ is equivalent to:

$$
I^{*}(N)=\operatorname{Var}(\widetilde{v} / w)^{-1} \underset{N \rightarrow+\infty}{\sim} \frac{2}{\sigma_{v}^{2}} .
$$

Proof : The result is obtained simply as a corollary of proposition 5.4 and where $\sigma=\sigma^{*}(N)=$ $\sigma_{v} \sqrt{\frac{N-3}{2}}$ 


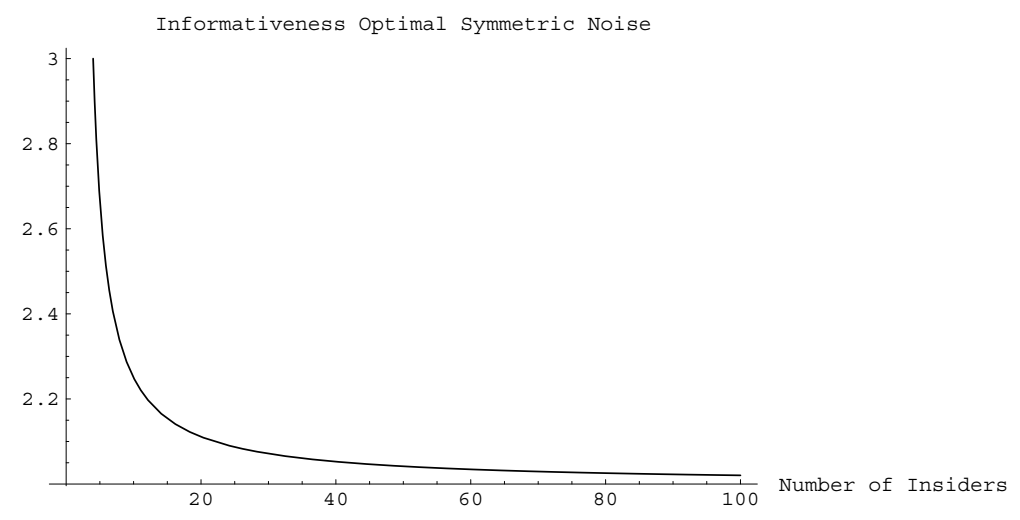

Figure 25: Informativeness Optimal Symmetric Noise.

Figures 25 and 26 represent the informativeness of prices respectively for the optimal level of noise $\sigma=\sigma^{*}(N)$ and for the perfect information $\sigma=0$ cases as functions of the number of insiders. While in the former case the informativeness of prices is decreasing with the number of insiders and has a finite positive limit $\frac{2}{\sigma_{v}^{2}}$, in the latter case it is increasing and tends to infinity with the number of insiders.

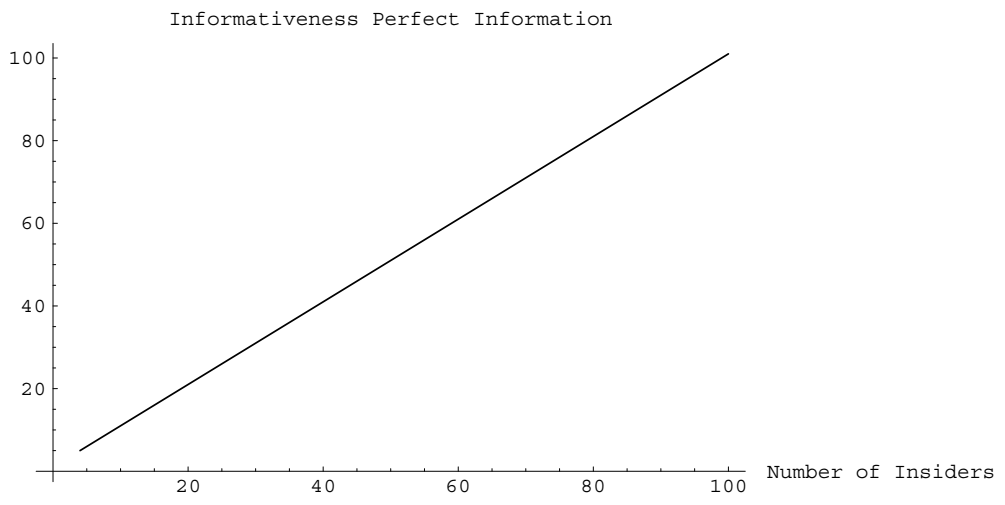

Figure 26: Informativeness Perfect Information.

Proposition 5.9 : At the equilibrium (in the symmetric case) and for the optimal level of noise $\sigma^{*}(N)$, the aggregate profits for each player are:

- $\pi_{\text {informed traders }}^{*}(N)=\pi^{*}(N)=\frac{\sigma_{v} \sigma_{u}}{2 \sqrt{2}} \frac{1}{\sqrt{1-\frac{1}{N}}}$,

- $\pi_{\text {noise traders }}^{*}(N)=-\pi^{*}(N)=-\frac{\sigma_{v} \sigma_{u}}{2 \sqrt{2}} \frac{1}{\sqrt{1-\frac{1}{N}}}$

- $\pi_{\text {market makers }}^{*}=0$. 
Therefore when $N$ is large the noise traders make on average the following loss: $-\frac{\sigma_{v} \sigma_{u}}{2 \sqrt{2}}$.

Proof : Since this is a zero-sum game and since the market makers perform a zero profit, the result is straightforward. 


\section{Concluding Remarks}

The main messages of this paper are threefold.

- The general study of $\mathrm{N}$ informed traders endowed with noisy signals and competing à la Nash in a market was still an issue that we have solved. We have shown the existence and uniqueness of a linear equilibrium.

- We have exhibited and delineated regions for which each informed traders is better off with respect to the perfectly informed case. The aggregate profit has a finite (strictly) positive limit when $\mathrm{N}$ is large. Therefore, even when an infinite number of insiders compete in the market the price is no longer efficient.

- In the particular situation in which each informed agent is endowed with a signal of which precision is the same, we have shown the existence of an optimal level of noise maximizing each individual profit. In the latter case, the liquidity is an increasing function of the number of informed traders but has a finite limit for large $\mathrm{N}$ and the informativeness of prices is a decreasing function of the number of informed traders. We also stress that for each given level of precision there exists an optimal size $N^{*}(\sigma)$ of the market that maximizes the aggregate expected profit. 


\section{References}

Admati, A. and P. Pfleiderer (1987), "A Viable Allocation of Information", Journal of Economic Theory, 43, 76-115.

Admati, A. and P. Pfleiderer (1988a), "Selling and Trading on Information in Financial Markets", American Economic Review, 78, 1165-1183.

Admati, A. and P. Pfleiderer (1988b), "A Theory of Intraday Patterns: Volume and Price Variability", The Review of Financial Studies, 1, 3-40.

Back, K. (1995), "Imperfect Competition Among Informed Traders", Forthcoming Journal of Finance.

Biais, B. and L. Germain (1997), "Incentive Compatible Contracts for the Sale of Information", mimeo London Business School.

Biais, B., D. Martimort and J. C. Rochet (1997), "Competing Mechanisms in a Common Value Environment", mimeo Université de Toulouse.

Dridi, R. and L. Germain (1999a), "Bullish Bearish Strategies of Trading: a Nonlinear Equilibrium", mimeo London Business School, London School of Economics.

Fishman, M. and K. Hagerty (1995), "The Incentives to Sell Financial Market Information", Journal of Financial Intermediation, 4, 95-115.

Foster, F. D. and S. Viswanathan (1994), "Strategic Trading with Asymmetrically Informed Traders and Long-lived Information", Journal of Financial and Quantitative Analysis, 29, 499-518.

Foster, F. D. and S. Viswanathan (1996), "Strategic Trading When Agents Forecast the Forecasts of Others", Journal of Finance, 51, 1437-78.

Germain, L. (1998), "Strategic Noise in Competitive Markets for the Sale of Information", mimeo London Business School.

Grossman, S. J. (1976), "On the Efficiency of Competitive Stock Markets Where Traders Have Diverse Information", Journal of Finance, 31, 573-585.

Grossman, S. J. and J. E. Stiglitz (1980), "On the Impossibility of Informationally Efficient Markets", American Economic Review, 70, 393-408.

Holden, C. W. and A. Subrahmanyam (1992), "Long-Lived Private Information and Imperfect Competition", The Journal of Finance, 47, 247-270.

Holden, C. W. and A. Subrahmanyam (1994), "Risk Aversion, Imperfect Competition and Long-lived Information", Economics Letters, 44, 181-190. 
Kyle, A. (1984), "Market Structure, Information, Futures Markets and Price Formation in International Agricultural Trade", Advanced Reading in Price Formation, Market Structure, and Price Instability ed. Story, Schmitz and Sarris (Westview Press, Boulder and London), 45-64.

Kyle, A. (1985), "Continuous Auctions and Insider Trading", Econometrica, 53, 1315-1335.

Rochet, J-C. and J-L. Villa (1994), "Insider Trading without Normality", Review of Economic Studies, 61,131-152.

Simonov, A. (1999), "Competition in Information Markets", Mimeo INSEAD.

Vives, X. (1995), "The Speed of Information Revelation in a Financial Market Mechanism", Journal of Economic Theory, 67, 178-204. 


\section{Appendices}

\section{A.1. Proof of propositions $3.1-3.2$}

The agent maximizes his conditional expected profit:

$$
\max _{x \in \mathbb{R}} E\{x[\widetilde{v}-\lambda(x+\widetilde{u})] / S\} .
$$

Since $\widetilde{u} \Perp \widetilde{S}$, we have:

$$
\begin{aligned}
& \Longleftrightarrow \max _{x \in \mathbb{R}} E\{x[\widetilde{v}-\lambda x] / S\}, \\
& \Longleftrightarrow \max _{x \in \mathbb{R}} x\left[\frac{S}{1+\tau}-\lambda x\right] .
\end{aligned}
$$

The necessary and sufficient first order conditions give:

$$
\widetilde{x}=\frac{1}{2 \lambda} \frac{1}{1+\tau} S=\beta S .
$$

Thus, the informed agent's best response is linear in his signal. Given that, at equilibrium, $\widetilde{p}=E[\widetilde{v} / w]$, we have thanks to the aforementioned linearity in the trading strategy $\widetilde{x}$ and the normality assumption that $\widetilde{p}=\lambda w$ is fulfilled and:

$$
\begin{aligned}
& \lambda=\frac{\operatorname{Cov}(\widetilde{v}, \widetilde{w})}{\operatorname{Var}(\widetilde{w})}=\frac{\operatorname{Cov}(\widetilde{v}, \beta \widetilde{S}+\widetilde{u})}{\operatorname{Var}(\beta \widetilde{S}+\widetilde{u})}=\frac{\beta \sigma_{v}^{2}}{\beta^{2}\left(\sigma_{v}^{2}+\sigma_{\varepsilon}^{2}\right)+\sigma_{u}^{2}}, \\
& \frac{1}{\lambda}=\beta(1+\tau)+\frac{\sigma_{u}^{2}}{\sigma_{v}^{2}} \frac{1}{\beta} .
\end{aligned}
$$

We thus have:

$$
\begin{aligned}
& \text { - } \frac{1}{\lambda}=\beta(1+\tau)+\frac{\sigma_{u}^{2}}{\sigma_{v}^{2}} \frac{1}{\beta}, \\
& \text { - } \frac{1}{\lambda}=2 \beta(1+\tau) \cdot
\end{aligned}
$$

We easily deduce that:

$$
\begin{aligned}
& \text { - } \beta^{*}(\sigma)=\frac{\sigma_{u}}{\sigma_{v}} \frac{1}{\sqrt{1+\tau}}, \\
& \text { - } \frac{1}{\lambda^{*}}(\sigma)=2 \frac{\sigma_{u}}{\sigma_{v}} \sqrt{1+\tau} .
\end{aligned}
$$

This ends the proof of proposition 3.1. The expected profit $\pi^{*}(\sigma)$ is given by:

$$
\begin{aligned}
& \pi^{*}(\sigma)=E\left\{\beta^{*}(\sigma) \widetilde{S}\left[\widetilde{v}-\lambda^{*}(\sigma) \widetilde{w}\right]\right\} \\
& \pi^{*}(\sigma)=\beta^{*}(\sigma) \sigma_{v}^{2}-\beta^{*^{2}}(\sigma) \lambda^{*}(\sigma)\left(\sigma_{v}^{2}+\sigma_{\varepsilon}^{2}\right), \\
& \pi^{*}(\sigma)=\sigma_{u} \sigma_{v} \frac{1}{\sqrt{1+\tau}}-\frac{1}{2(1+\tau)}(1+\tau) \frac{1}{\sqrt{1+\tau}} \sigma_{u} \sigma_{v}, \\
& \pi^{*}(\sigma)=\frac{1}{2} \sigma_{u} \sigma_{v} \frac{1}{\sqrt{1+\tau}} .
\end{aligned}
$$


This ends the proof of proposition 3.2

\section{A.2. Proof of propositions $3.3-3.4$}

Agent $i$ maximizes his conditional expected profit $(N \geq 2)$ :

$$
\max _{x \in \mathbb{R}} E\left\{x\left[\widetilde{v}-\lambda\left(x+\sum_{j \neq i} \widetilde{x}_{j}+\widetilde{u}\right)\right] / S_{i}\right\}, i=1, \ldots, N
$$

since $\widetilde{u} \Perp \widetilde{S}_{i}$, we have:

$$
\begin{gathered}
\Longleftrightarrow \max _{x \in \mathbb{R}} E\left\{x\left[\widetilde{v}-\lambda\left(x+\sum_{j \neq i} \widetilde{x}_{j}\right)\right] / S_{i}\right\}, i=1, \ldots, N, \\
\max _{x \in \mathbb{R}} x\left\{\frac{1}{1+\tau_{i}} S_{i}-\lambda\left[x+\sum_{j \neq i} E\left(\widetilde{x}_{j} / S_{i}\right)\right]\right\}, i=1, \ldots, N, \\
\max _{x \in \mathbb{R}} \frac{1}{1+\tau_{i}} S_{i} x-\lambda x^{2}-\lambda x \sum_{j \neq i} E\left(\widetilde{x}_{j} / S_{i}\right), i=1, \ldots, N .
\end{gathered}
$$

The necessary and sufficient first order conditions are:

$$
\begin{array}{r}
\frac{1}{1+\tau_{i}} S_{i}-2 \lambda \widetilde{x}_{i}-\lambda \sum_{j \neq i} E\left(\widetilde{x}_{j} / S_{i}\right)=0, i=1, \ldots, N, \\
\Longleftrightarrow \widetilde{x}_{i}=\frac{1}{2 \lambda} \frac{1}{1+\tau_{i}} S_{i}-\frac{1}{2} \sum_{j \neq i} E\left(\widetilde{x}_{j} / S_{i}\right)=0, i=1, \ldots, N,
\end{array}
$$

Let $\left\{x_{i}^{*}, i=1, \ldots, N\right\}$ be a particular solution to (6.1). Then if we define:

$$
\widetilde{y}_{i}=\widetilde{x}_{i}-x_{i}^{*}, i=1, \ldots, N,
$$

$\left\{\widetilde{x}_{i}, i=1, \ldots, N\right\}$ is solution to $(6.1)$ if and only if $\left\{\widetilde{y}_{i}, i=1, \ldots, N\right\}$ is solution to $(6.2)$ :

$$
\widetilde{y}_{i}=-\frac{1}{2} \sum_{j \neq i} E\left(\widetilde{y}_{j} / S_{i}\right), i=1, \ldots, N
$$

Remark 6.1 : We will in fact show that there exists a particular linear solution $x_{i}^{*}=\beta_{i} S_{i}, i=$ $1, \ldots, N$.

Remark 6.2 : We will show that the set of solution (6.2) is reduced to the singleton $0 \in \mathbb{R}^{N}$, that is, $\left\{\widetilde{y}_{i}, i=1, \ldots, N\right\}$ is solution to (6.2) if and only if $\widetilde{y}_{i} \stackrel{a . s .}{=} 0, i=1, \ldots, N$.

$$
(6.2) \Longrightarrow\left\{\begin{array}{l}
\widetilde{y}_{i}+\frac{1}{2} \sum_{j \neq i} \widetilde{y}_{j}=\widetilde{\eta}_{i}, \\
\text { with } \widetilde{\eta}_{i} \perp \sigma\left(S_{i}\right) \Longleftrightarrow E\left(\widetilde{\eta}_{i} / S_{i}\right)=0 .
\end{array}\right.
$$


We introduce:

$$
\Sigma=\left[\begin{array}{ccccccc}
1 & \frac{1}{2} & \frac{1}{2} & \ldots & \frac{1}{2} & \ldots & \frac{1}{2} \\
\frac{1}{2} & 1 & \frac{1}{2} & \ldots & \frac{1}{2} & \ldots & \frac{1}{2} \\
\frac{1}{2} & \frac{1}{2} & 1 & \ldots & \frac{1}{2} & \ldots & \frac{1}{2} \\
\vdots & \vdots & \vdots & \ddots & \vdots & \vdots & \vdots \\
\frac{1}{2} & \frac{1}{2} & \frac{1}{2} & \ldots & 1 & \ldots & \frac{1}{2} \\
\vdots & \vdots & \vdots & \vdots & \vdots & \ddots & \vdots \\
\frac{1}{2} & \frac{1}{2} & \frac{1}{2} & \ldots & \frac{1}{2} & \ldots & 1
\end{array}\right],
$$

the $N \times N$ matrix. It is easy to show that $\Sigma$ is not singular and that:

$$
\Sigma^{-1}=\frac{2}{N+1}\left[\begin{array}{llclclc}
N & -1 & -1 & \ldots & -1 & \ldots & -1 \\
-1 & N & -1 & \ldots & -1 & \ldots & -1 \\
-1 & -1 & N & \ldots & -1 & \ldots & -1 \\
\vdots & \vdots & \vdots & \ddots & \vdots & \vdots & \vdots \\
-1 & -1 & -1 & \ldots & N & \ldots & -1 \\
\vdots & \vdots & \vdots & \vdots & \vdots & \ddots & \vdots \\
-1 & -1 & -1 & \ldots & -1 & \ldots & N
\end{array}\right] .
$$

We denote $\widetilde{y}=\left(\widetilde{y}_{1}, \ldots, \widetilde{y}_{N}\right)^{\prime}$ and $\widetilde{\eta}=\left(\widetilde{\eta}_{1}, \ldots, \widetilde{\eta}_{N}\right)^{\prime} . \quad(6.2) \Longrightarrow \Sigma \widetilde{y}=\widetilde{\eta}$, that is $\widetilde{y}=\Sigma^{-1} \widetilde{\eta}$. Therefore, for $i=1, \ldots, N$, we have:

$$
\widetilde{y}_{i}=\frac{2}{N+1}\left\{N \widetilde{\eta}_{i}-\sum_{j \neq i} \widetilde{\eta}_{j}\right\}
$$

If we plug the latter relation into (6.2), we obtain $\widetilde{y}$ is solution to (6.2) if and only if there exist $\left\{\widetilde{\eta}_{i}, i=1, \ldots, N\right\}$ such that $\widetilde{\eta}_{i} \perp \sigma\left(S_{i}\right)$ and:

(1) $\widetilde{y}_{i}=\frac{2}{N+1}\left\{N \widetilde{\eta}_{i}-\sum_{j \neq i} \widetilde{\eta}_{j}\right\}, i=1, \ldots, N$,

(2) $\frac{2}{N+1}\left\{N \widetilde{\eta}_{i}-\sum_{j \neq i} \widetilde{\eta}_{j}\right\}=-\frac{1}{2} \sum_{j \neq i} E\left\{\frac{2}{N+1}\left[N \widetilde{\eta}_{j}-\sum_{k \neq j} \widetilde{\eta}_{k}\right] / S_{i}\right\}$,

$\Longleftrightarrow$ (1) and $N \widetilde{\eta}_{i}-\sum_{j \neq i} \widetilde{\eta}_{j}=-\frac{1}{2} E\left\{N \sum_{j \neq i} \widetilde{\eta}_{j}-\sum_{j \neq i} \sum_{k \neq j} \widetilde{\eta}_{k} / S_{i}\right\}$,

$\Longleftrightarrow$ (1) and $N \widetilde{\eta}_{i}-\sum_{j \neq i} \widetilde{\eta}_{j}=-\frac{1}{2} E\left\{N \sum_{j \neq i} \widetilde{\eta}_{j}-(N-1) \widetilde{\eta}_{i}-(N-2) \sum_{k \neq i} \widetilde{\eta}_{k} / S_{i}\right\}$,

indeed $\sum_{j \neq i} \sum_{k \neq j} \widetilde{\eta}_{k}=\sum_{j \neq i}\left[\sum_{k=1}^{N} \widetilde{\eta}_{k}-\widetilde{\eta}_{j}\right]=(N-1) \sum_{k=1}^{N} \widetilde{\eta}_{k}-\sum_{j \neq i} \widetilde{\eta}_{j}=(N-1) \widetilde{\eta}_{i}+(N-1) \sum_{k \neq i} \widetilde{\eta}_{k}-\sum_{j \neq i} \widetilde{\eta}_{j}=$ $(N-1) \widetilde{\eta}_{i}+(N-2) \sum_{k \neq i} \widetilde{\eta}_{k}(N \geq 2)$. Thus $\widetilde{y}$ is solution to (6.2) if and only if there exist 
$\left\{\widetilde{\eta}_{i}, i=1, \ldots, N\right\}$ such that $\widetilde{\eta}_{i} \perp \sigma\left(S_{i}\right)$ and:

$$
\begin{aligned}
& \widetilde{y}_{i}=\frac{2}{N+1}\left\{N \widetilde{\eta}_{i}-\sum_{j \neq i} \widetilde{\eta}_{j}\right\}, i=1, \ldots, N, \\
& N \widetilde{\eta}_{i}-\sum_{j \neq i} \widetilde{\eta}_{j}=-E\left[\sum_{j \neq i} \widetilde{\eta}_{j} / S_{i}\right],
\end{aligned}
$$

since $E\left[\widetilde{\eta}_{i} / S_{i}\right]=0$. We have $N \geq 2$ :

$$
N \widetilde{\eta}_{i}=\sum_{j \neq i} \widetilde{\eta}_{j}-E\left[\sum_{j \neq i} \widetilde{\eta}_{j} / S_{i}\right], i=1, \ldots, N
$$

From the latter equation, we deduce that $E\left(\widetilde{\eta}_{i}\right)=0, i=1, \ldots, N$ since $E\left(\widetilde{\eta}_{i}\right)=$ $\frac{1}{N} E\left\{\widetilde{z}-E\left[\widetilde{z} / S_{i}\right]\right\}=0$ where $\widetilde{z}=\sum_{j \neq i} \widetilde{\eta}_{j}$.

$$
\begin{aligned}
\operatorname{Var}\left(\widetilde{\eta}_{i}\right) & =\frac{1}{N^{2}} \operatorname{Var}\left\{\widetilde{z}-E\left[\widetilde{z} / S_{i}\right]\right\}=\frac{1}{N^{2}} E\left[\operatorname{Var}\left(\widetilde{z} / S_{i}\right)\right] \\
& \leq \frac{1}{N^{2}} \operatorname{Var}(\widetilde{z})=\frac{1}{N^{2}} \operatorname{Var}\left(\sum_{j \neq i} \widetilde{\eta}_{j}\right) \leq \frac{1}{N^{2}}\left[\sum_{j \neq i} \sqrt{\operatorname{Var}\left(\widetilde{\eta}_{j}\right)}\right]^{2}
\end{aligned}
$$

Indeed $\operatorname{Var}\left(\sum_{j \neq i} \widetilde{\eta}_{j}\right)=\sum_{j \neq i} \operatorname{Var}\left(\widetilde{\eta}_{j}\right)+2 \sum_{j, k \neq i} \operatorname{Cov}\left(\widetilde{\eta}_{j}, \widetilde{\eta}_{k}\right) \leq \sum_{j \neq i}\left[\sqrt{\operatorname{Var}\left(\widetilde{\eta}_{j}\right)}\right]^{2}+$ $k<j$

$+2 \sum_{\substack{j, k \neq i \\ k<j}} \sqrt{\operatorname{Var}\left(\widetilde{\eta}_{j}\right)} \sqrt{\operatorname{Var}\left(\widetilde{\eta}_{k}\right)}=\left[\sum_{j \neq i} \sqrt{\operatorname{Var}\left(\widetilde{\eta}_{j}\right)}\right]^{2}$.

$$
\begin{aligned}
& \Longrightarrow \quad \forall i=1, \ldots, N, \operatorname{Var}\left(\widetilde{\eta}_{i}\right) \leq\left(\frac{N-1}{N}\right)^{2} \max _{j=1, \ldots, N}\left\{\operatorname{Var}\left(\widetilde{\eta}_{j}\right)\right\} \\
& \Longrightarrow \max _{i=1, \ldots, N} \operatorname{Var}\left(\widetilde{\eta}_{i}\right) \leq\left(\frac{N-1}{N}\right)^{2} \max _{j=1, \ldots, N}\left\{\operatorname{Var}\left(\widetilde{\eta}_{j}\right)\right\} \\
& \Longrightarrow\left[1-\left(1-\frac{1}{N}\right)^{2}\right] \underset{i=1, \ldots, N}{\max _{1} \operatorname{Var}\left(\widetilde{\eta}_{i}\right) \leq 0,} \\
& \Longrightarrow \max _{i=1, \ldots, N} \operatorname{Var}\left(\widetilde{\eta}_{i}\right)=0 .
\end{aligned}
$$

Thus we have for $i=1, \ldots, N$ :

$$
\left.\begin{array}{l}
E\left(\widetilde{\eta}_{i}\right)=0, \\
\operatorname{Var}\left(\widetilde{\eta}_{i}\right)=0,
\end{array}\right\} \Longrightarrow \widetilde{\eta}_{i} \stackrel{a . s .}{=} 0
$$


Therefore the only solution to $(6.2)$ is $\widetilde{y}=0 \in \mathbb{R}^{N}$.

We now show the existence of a linear equilibrium $\left\{x_{i}^{*}, i=1, \ldots, N\right\}$ which is, in addition, linear in the information. Hence and from the previous property, it corresponds to the unique linear equilibrium. We thus look for a particular solution to (6.2) of the form: $\left\{x_{i}^{*}=\beta_{i}^{*} S_{i}, i=1, \ldots, N\right\}$. We introduce the following quantities:

$$
\begin{aligned}
& a_{i}=\frac{1}{1+\tau_{i}}<1, i=1, \ldots, N, \\
& b_{i}=\frac{1}{2 \lambda} a_{i} .
\end{aligned}
$$

Thanks to the normality assumption and the mutual independence of $\left\{\widetilde{v}, \widetilde{\varepsilon}_{1}, \ldots, \widetilde{\varepsilon}_{N}\right\}$, we have $E\left(\widetilde{S}_{j} / S_{i}\right)=a_{i} S_{i}$ for $j \neq i$. Using (6.1), we have:

$$
\begin{aligned}
& \beta_{i} S_{i}=b_{i} S_{i}-\frac{1}{2} \sum_{j \neq i} E\left[\beta_{j} \widetilde{S}_{j} / S_{i}\right], i=1, \ldots, N, \\
& \beta_{i} S_{i}=b_{i} S_{i}-\frac{1}{2} \sum_{j \neq i} a_{i} \beta_{j} \widetilde{S}_{j}, i=1, \ldots, N,
\end{aligned}
$$

or equivalently:

$$
\begin{gathered}
\beta_{i}=b_{i}-\frac{a_{i}}{2} \sum_{j \neq i} \beta_{j}, i=1, \ldots, N, \\
\beta_{i}\left(1-\frac{a_{i}}{2}\right)=b_{i}-\frac{a_{i}}{2} \sum_{j=1}^{N} \beta_{j}, i=1, \ldots, N, \\
\beta_{i}\left(\frac{2-a_{i}}{2 a_{i}}\right)=\frac{1}{2 \lambda}-\frac{1}{2} \sum_{j=1}^{N} \beta_{j}, i=1, \ldots, N, \\
\beta_{i}\left(\frac{1}{2}+\tau_{i}\right)=\frac{1}{2 \lambda}-\frac{1}{2} \sum_{j=1}^{N} \beta_{j}, i=1, \ldots, N, \\
\beta_{i}=\frac{a}{1+2 \tau_{i}}, \\
\frac{1}{\lambda}=a\left[1+\sum_{j=1}^{N} \frac{1}{1+2 \tau_{j}}\right],
\end{gathered}
$$

with $a$ independent of $\mathrm{i}$. We also know that, at equilibrium, $\widetilde{p}=E(\widetilde{v} / w)$. In this particular equilibrium, we have $\widetilde{w}=\sum_{i=1}^{N} \beta_{i} \widetilde{S}_{i}+\widetilde{u}$. Thanks to the mutual independence of $\left\{\widetilde{v}, \widetilde{\varepsilon}_{1}, \ldots, \widetilde{\varepsilon}_{N}, \widetilde{u}\right\}$ and normality of each components, we deduce that $\widetilde{p}=\lambda w$ holds and that therefore we do 
obtain a linear equilibrium with:

$$
\begin{aligned}
& \lambda=\frac{\operatorname{Cov}(\widetilde{v}, \widetilde{w})}{\operatorname{Var}(\widetilde{w})}=\frac{\operatorname{Cov}\left(\widetilde{v}, \sum_{j=1}^{N} \beta_{j} \widetilde{S}_{j}+\widetilde{u}\right)}{\operatorname{Var}\left(\sum_{j=1}^{N} \beta_{j} \widetilde{S}_{j}+\widetilde{u}\right)}, \\
& \lambda=\frac{\sum_{j=1}^{N} \beta_{j} \sigma_{v}^{2}}{\operatorname{Var}_{\left(\sum_{j=1}^{N} \beta_{j} \widetilde{v}+\sum_{j=1}^{N} \beta_{j} \widetilde{\varepsilon}_{j}+\widetilde{u}\right)}=\frac{\sum_{j=1}^{N} \beta_{j} \sigma_{v}^{2}}{\left(\sum_{j=1}^{N} \beta_{j}\right)^{2} \sigma_{v}^{2}+\sum_{j=1}^{N} \beta_{j}^{2} \sigma_{j}^{2}+\sigma_{u}^{2}}}, \\
& \frac{1}{\lambda}=\sum_{j=1}^{N} \beta_{j}+\frac{\sum_{j=1}^{N} \beta_{j}^{2} \tau_{j}}{\sum_{j=1}^{N} \beta_{j}}+\frac{\sigma_{u}^{2}}{\sigma_{v}^{2}} \frac{1}{\sum_{j=1}^{N} \beta_{j}} \cdot \\
& \frac{1}{\lambda}=a \sum_{j=1}^{N} \frac{1}{1+2 \tau_{j}}+a \frac{\sum_{j=1}^{N} \tau_{j} \frac{1}{\left(1+2 \tau_{j}\right)^{2}}}{\sum_{j=1}^{N} \frac{1}{1+2 \tau_{j}}}+\frac{\sigma_{u}^{2}}{\sigma_{v}^{2}} \frac{1}{a \sum_{j=1}^{N} \frac{1}{1+2 \tau_{j}}} .
\end{aligned}
$$

Using (6.3) and (6.4), we obtain:

$$
\begin{aligned}
& a\left\{1-\frac{\sum_{j=1}^{N} \frac{\tau_{j}}{\left(1+2 \tau_{j}\right)^{2}}}{\sum_{j=1}^{N} \frac{1}{1+2 \tau_{j}}}\right\}=\frac{\sigma_{u}^{2}}{\sigma_{v}^{2}} \frac{1}{a \sum_{j=1}^{N} \frac{1}{1+2 \tau_{j}}}, \\
& a^{2}\left\{\sum_{j=1}^{N} \frac{1}{1+2 \tau_{j}}-\sum_{j=1}^{N} \frac{\tau_{j}}{\left(1+2 \tau_{j}\right)^{2}}\right\}=\frac{\sigma_{u}^{2}}{\sigma_{v}^{2}}, \\
& a^{2} \sum_{j=1}^{N} \frac{1+\tau_{j}}{\left(1+2 \tau_{j}\right)^{2}}=\frac{\sigma_{u}^{2}}{\sigma_{v}^{2}}
\end{aligned}
$$




$$
\begin{aligned}
& a=\frac{\sigma_{u}}{\sigma_{v}} \frac{1}{\left\{\sum_{j=1}^{N} \frac{1+\tau_{j}}{\left(1+2 \tau_{j}\right)^{2}}\right\}^{\frac{1}{2}}}, \\
& \beta_{i}^{*}(\tau)=\frac{\sigma_{u}}{\sigma_{v}} \frac{1}{1+2 \tau_{i}} \frac{1}{\left\{\sum_{j=1}^{N} \frac{1+\tau_{j}}{\left(1+2 \tau_{j}\right)^{2}}\right\}^{\frac{1}{2}}}, \\
& \frac{1}{\lambda^{*}(\tau)}=\frac{\sigma_{u}}{\sigma_{v}} \frac{1}{\left\{\sum_{j=1}^{N} \frac{1+\tau_{j}}{\left(1+2 \tau_{j}\right)^{2}}\right\}^{\frac{1}{2}}\left[1+\sum_{j=1}^{N} \frac{1}{1+2 \tau_{j}}\right] .} .
\end{aligned}
$$

This ends the proof of proposition 3.3.

$$
\begin{aligned}
& \text { - } \pi_{i}^{*}(\sigma)=\beta_{i}^{*}\left(1-\lambda^{*} \sum_{j=1}^{N} \beta_{j}^{*}\right) \sigma_{v}^{2}-\lambda^{*} \beta_{i}^{*^{2}} \sigma_{i}^{2}=\sigma_{v}^{2}\left[\beta_{i}^{*}\left(1-\lambda^{*} \sum_{j=1}^{N} \beta_{j}^{*}\right)-\lambda^{*} \beta_{i}^{*^{2}} \tau_{i}\right], \\
& \text { - } \beta_{i}^{*}(\sigma)=\frac{a}{1+2 \tau_{i}}, \quad a=\frac{\sigma_{u}}{\sigma_{v}} \frac{1}{\left\{\sum_{j=1}^{N} \frac{1+\tau_{j}}{\left(1+2 \tau_{j}\right)^{2}}\right\}^{\frac{1}{2}}}, \\
& \text { - } \lambda^{*}(\sigma)=\frac{1}{a} \frac{1}{1+\sum_{j=1}^{N} \frac{1}{1+2 \tau_{j}}}, \quad \lambda^{*}(\sigma) \beta_{i}^{*}(\sigma)=\frac{1}{1+2 \tau_{i}} \frac{1}{1+\sum_{j=1}^{N} \frac{1}{1+2 \tau_{j}}}, \\
& \text { - } \quad \sum_{j=1}^{N} \lambda^{*} \beta_{i}^{*}(\sigma)=1-\frac{1}{1+\sum_{j=1}^{N} \frac{1}{1+2 \tau_{j}}} \\
& \Longrightarrow \pi_{i}^{*}(\sigma)=\sigma_{v}^{2}\left\{\frac{a}{1+2 \tau_{i}} \frac{1}{1+\sum_{j=1}^{N} \frac{1}{1+2 \tau_{j}}}-\frac{a}{\left(1+2 \tau_{i}\right)^{2}} \frac{\tau_{i}}{1+\sum_{j=1}^{N} \frac{1}{1+2 \tau_{j}}}\right\}, \\
& \pi_{i}^{*}(\sigma)=\frac{\sigma_{v}^{2} a}{\left(1+2 \tau_{i}\right)\left[1+\sum_{j=1}^{N} \frac{1}{1+2 \tau_{j}}\right]}\left\{1-\frac{\tau_{i}}{1+2 \tau_{i}}\right\}=\frac{\sigma_{v}^{2} a}{\left(1+2 \tau_{i}\right)\left[1+\sum_{j=1}^{N} \frac{1}{1+2 \tau_{j}}\right]} \frac{1+\tau_{i}}{1+2 \tau_{i}},
\end{aligned}
$$

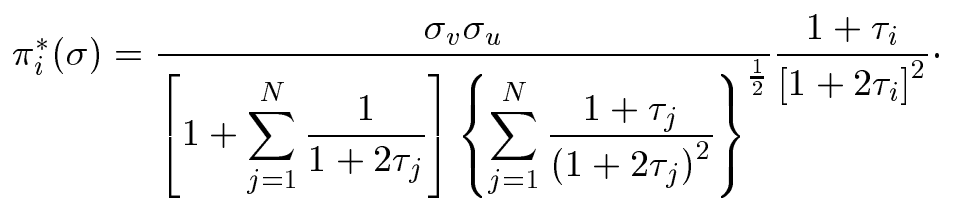


The aggregate profit is straightforwardly deduced. This ends the proof of proposition 3.4.

\section{B.1. Proof of proposition 4.1}

$$
\begin{aligned}
& \frac{\partial \beta_{i}^{*}}{\partial \tau_{j}}(\tau)=\frac{1}{2} \frac{\sigma_{u}}{\sigma_{v}} \frac{3+2 \tau_{j}}{\left\{\sum_{j=1}^{N} \frac{1+\tau_{j}}{\left(1+2 \tau_{j}\right)^{2}}\right\}^{\frac{3}{2}}\left(1+2 \tau_{i}\right)\left(1+2 \tau_{j}\right)^{3}}>0, \text { for } j \neq i \\
& \frac{\partial \beta_{i}^{*}}{\partial \tau_{i}}(\tau)=-\frac{1}{2} \frac{\sigma_{u}}{\sigma_{v}} \frac{4\left(1+2 \tau_{i}\right) \sum_{j \neq i} \frac{1+\tau_{j}}{\left(1+2 \tau_{j}\right)^{2}}+1}{\left\{\sum_{j=1}^{N} \frac{1+\tau_{j}}{\left(1+2 \tau_{j}\right)^{2}}\right\}^{\frac{3}{2}}\left(1+2 \tau_{i}\right)^{3}}<0 .
\end{aligned}
$$

Indeed:

$$
\begin{aligned}
& \frac{\partial \beta_{i}^{*}}{\partial \tau_{i}}(\tau)=\frac{1}{2} \frac{\sigma_{u}}{\sigma_{v}} \frac{3+2 \tau_{i}}{\left\{\sum_{j=1}^{N} \frac{1+\tau_{j}}{\left(1+2 \tau_{j}\right)^{2}}\right\}^{\frac{3}{2}}\left(1+2 \tau_{i}\right)^{4}}-\frac{\sigma_{u}}{\sigma_{v}} \frac{2}{\left\{\sum_{j=1}^{N} \frac{1+\tau_{j}}{\left(1+2 \tau_{j}\right)^{2}}\right\}^{\frac{1}{2}}\left(1+2 \tau_{i}\right)^{2}}, \\
& \left.\frac{\partial \beta_{i}^{*}}{\partial \tau_{i}}\right)(\tau)=\frac{1}{2} \frac{\sigma_{u}}{\sigma_{v}} \frac{3+2 \tau_{i}-4\left(1+2 \tau_{i}\right)^{2} \sum_{j=1}^{N} \frac{1+\tau_{j}}{\left(1+2 \tau_{j}\right)^{2}}}{\left\{\sum_{j=1}^{N} \frac{1+\tau_{j}}{\left(1+2 \tau_{j}\right)^{2}}\right\}^{\frac{3}{2}}\left(1+2 \tau_{i}\right)^{4}}=-\frac{1}{2} \frac{\sigma_{u}}{\sigma_{v}} \frac{4\left(1+2 \tau_{i}\right)^{2} \sum_{j=1}^{N} \frac{1+\tau_{j}}{\left(1+2 \tau_{j}\right)^{2}}+1+2 \tau_{i}}{\left\{\sum_{j=1}^{N} \frac{1+\tau_{j}}{\left(1+2 \tau_{j}\right)^{2}}\right\}^{\frac{3}{2}}\left(1+2 \tau_{i}\right)^{4}}, \\
& \left.\frac{\partial \beta_{i}^{*}}{\partial \tau_{i}}\right)(\tau)=-\frac{1}{2} \frac{\sigma_{u}}{\sigma_{v}} \frac{4\left(1+2 \tau_{i}\right) \sum_{j \neq i} \frac{1+\tau_{j}}{\left(1+2 \tau_{j}\right)^{2}}+1}{\left\{\sum_{j=1}^{N} \frac{1+\tau_{j}}{\left(1+2 \tau_{j}\right)^{2}}\right\}^{\frac{3}{2}}\left(1+2 \tau_{i}\right)^{3}} .
\end{aligned}
$$

This ends the proof of proposition 4.1.

\section{B.2. Proof of proposition 4.2}

$$
\frac{\partial \lambda^{*^{-1}}}{\partial \tau_{i}}(\tau)=\frac{\sigma_{u}}{\sigma_{v}}\left\{\frac{1}{2} \frac{\left(3+2 \tau_{i}\right)\left[1+\sum_{j=1}^{N} \frac{1}{1+2 \tau_{j}}\right]}{\left\{\sum_{j=1}^{N} \frac{1+\tau_{j}}{\left(1+2 \tau_{j}\right)^{2}}\right\}^{\frac{3}{2}}\left(1+2 \tau_{i}\right)^{3}}-\frac{2}{\left\{\sum_{j=1}^{N} \frac{1+\tau_{j}}{\left(1+2 \tau_{j}\right)^{2}}\right\}^{\frac{1}{2}}\left(1+2 \tau_{i}\right)^{2}}\right\},
$$




$$
\begin{aligned}
& \frac{\partial \lambda^{*^{-1}}}{\partial \tau_{i}}(\tau)=\frac{1}{2} \frac{\sigma_{u}}{\sigma_{v}} \frac{\left\{\left(3+2 \tau_{i}\right)\left[1+\sum_{j=1}^{N} \frac{1}{1+2 \tau_{j}}\right]-4\left(1+2 \tau_{i}\right) \sum_{j=1}^{N} \frac{1+\tau_{j}}{\left(1+2 \tau_{j}\right)^{2}}\right\}}{\left\{\sum_{j=1}^{N} \frac{1+\tau_{j}}{\left(1+2 \tau_{j}\right)^{2}}\right\}^{\frac{3}{2}}\left(1+2 \tau_{i}\right)^{3}}, \\
& \frac{\partial \lambda^{*^{-1}}}{\partial \tau_{i}}(\tau)=\frac{1}{2} \frac{\sigma_{u}}{\sigma_{v}} \frac{1}{\left\{\sum_{j=1}^{N} \frac{1+\tau_{j}}{\left(1+2 \tau_{j}\right)^{2}}\right\}^{\frac{3}{2}}\left(1+2 \tau_{i}\right)^{3}} h_{i}(\tau),
\end{aligned}
$$

with : $h_{i}(\tau)=3+2 \tau_{i}+\frac{3+2 \tau_{i}}{1+2 \tau_{i}}+\left(3+2 \tau_{i}\right) \sum_{j \neq i} \frac{1}{1+2 \tau_{j}}-4 \frac{1+\tau_{i}}{1+2 \tau_{i}}-4\left(1+2 \tau_{i}\right) \sum_{j \neq i} \frac{1+\tau_{j}}{\left(1+2 \tau_{j}\right)^{2}}$,

$$
\begin{aligned}
& h_{i}(\tau)=2+2 \tau_{i}+\left(3+2 \tau_{i}\right) \sum_{j \neq i} \frac{1}{1+2 \tau_{j}}-4\left(1+2 \tau_{i}\right) \sum_{j \neq i} \frac{1+\tau_{j}}{\left(1+2 \tau_{j}\right)^{2}}, \\
& h_{i}(\tau)=\tau_{i}\left(2+2 \sum_{j \neq i} \frac{1}{1+2 \tau_{j}}-8 \sum_{j \neq i} \frac{1+\tau_{j}}{\left(1+2 \tau_{j}\right)^{2}}\right)+2+3 \sum_{j \neq i} \frac{1}{1+2 \tau_{j}}-4 \sum_{j \neq i} \frac{1+\tau_{j}}{\left(1+2 \tau_{j}\right)^{2}}, \\
& h_{i}(\tau)=2 \tau_{i}\left[1-\sum_{j \neq i} \frac{3+2 \tau_{j}}{\left(1+2 \tau_{j}\right)^{2}}\right]+2+\sum_{j \neq i} \frac{2 \tau_{j}-1}{\left(1+2 \tau_{j}\right)^{2}},
\end{aligned}
$$

We define $b\left(\tau_{-i}\right)=1-\sum_{j \neq i} \frac{3+2 \tau_{j}}{\left(1+2 \tau_{j}\right)^{2}}, c\left(\tau_{-i}\right)=2+\sum_{j \neq i} \frac{2 \tau_{j}-1}{\left(1+2 \tau_{j}\right)^{2}}$. It is worth noticing that $c\left(\tau_{-i}\right)>b\left(\tau_{-i}\right)$, indeed $c\left(\tau_{-i}\right)-b\left(\tau_{-i}\right)=1+\sum_{j \neq i} \frac{2}{1+2 \tau_{j}}$. This ends the proof of proposition 4.2.

\section{B.3. Proof of proposition 4.3}

$$
\begin{aligned}
\frac{\partial \ln \pi_{i}^{*}}{\partial \tau_{j}}(\tau) & =\frac{\partial}{\partial \tau_{j}}\left\{-\frac{1}{2} \ln \left[\sum_{k=1}^{N} \frac{1+\tau_{k}}{\left(1+2 \tau_{k}\right)^{2}}\right]-\ln \left[1+\sum_{k=1}^{N} \frac{1}{1+2 \tau_{k}}\right]\right\}, \\
& =\frac{1}{2} \frac{3+2 \tau_{j}}{\sum_{k=1}^{N} \frac{1+\tau_{k}}{\left(1+2 \tau_{k}\right)^{2}}\left(1+2 \tau_{j}\right)^{3}}+\frac{2}{\left[1+\sum_{k=1}^{N} \frac{1}{1+2 \tau_{k}}\right]\left(1+2 \tau_{j}\right)^{2}}>0,
\end{aligned}
$$

$\Longrightarrow \quad \pi_{i}^{*}(\sigma)$ is increasing in $\sigma_{-i}$. 


$$
\begin{aligned}
\bullet \frac{\partial \ln \pi_{i}^{*}}{\partial \tau_{i}}(\tau) & =\frac{1}{2} \frac{3+2 \tau_{i}}{\left(1+2 \tau_{i}\right)^{3} \sum_{k=1}^{N} \frac{1+\tau_{k}}{\left(1+2 \tau_{k}\right)^{2}}}+\frac{2}{\left(1+2 \tau_{i}\right)^{2}\left[1+\sum_{k=1}^{N} \frac{1}{1+2 \tau_{k}}\right]}+\frac{1}{1+\tau_{i}}-\frac{4}{1+2 \tau_{i}}, \\
& =\frac{1}{2} \frac{3+2 \tau_{i}}{\left(1+2 \tau_{i}\right)^{3} \sum_{k=1}^{N} \frac{1+\tau_{k}}{\left(1+2 \tau_{k}\right)^{2}}}+\frac{2}{\left(1+2 \tau_{i}\right)^{2}\left[1+\sum_{k=1}^{N} \frac{1}{1+2 \tau_{k}}\right]}-\frac{3+2 \tau_{i}}{\left(1+2 \tau_{i}\right)\left(1+\tau_{i}\right)}, \\
& =\frac{1}{2\left(1+2 \tau_{i}\right)^{3}\left[\sum_{k=1}^{N} \frac{1+\tau_{k}}{\left(1+2 \tau_{k}\right)^{2}}\right]\left[1+\sum_{k=1}^{N} \frac{1}{1+2 \tau_{k}}\right]\left(1+\tau_{i}\right)} h_{i}(\tau),
\end{aligned}
$$

where:

$$
\begin{aligned}
h_{i}(\tau) & =\left(3+2 \tau_{i}\right)\left(1+\tau_{i}\right)\left[1+\sum_{k=1}^{N} \frac{1}{1+2 \tau_{k}}\right]+4\left(1+2 \tau_{i}\right)\left[\sum_{k=1}^{N} \frac{1+\tau_{k}}{\left(1+2 \tau_{k}\right)^{2}}\right]\left(1+\tau_{i}\right) \\
& -2\left(3+2 \tau_{i}\right)\left(1+2 \tau_{i}\right)^{2} \sum_{k=1}^{N} \frac{1+\tau_{k}}{\left(1+2 \tau_{k}\right)^{2}}\left[1+\sum_{k=1}^{N} \frac{1}{1+2 \tau_{k}}\right] \\
& =\left(3+2 \tau_{i}\right)\left(1+\tau_{i}\right)+\frac{\left(3+2 \tau_{i}\right)\left(1+\tau_{i}\right)}{1+2 \tau_{i}}+\left(3+2 \tau_{i}\right)\left(1+\tau_{i}\right) \sum_{k \neq i} \frac{1}{1+2 \tau_{k}}+4 \frac{\left(1+\tau_{i}\right)^{2}}{1+2 \tau_{i}} \\
& +4\left(1+2 \tau_{i}\right)\left(1+\tau_{i}\right) \sum_{k \neq i} \frac{1+\tau_{k}}{\left(1+2 \tau_{k}\right)^{2}}-2\left(3+2 \tau_{i}\right)\left(1+\tau_{i}\right)-2\left(3+2 \tau_{i}\right)\left(1+2 \tau_{i}\right)^{2} \sum_{k \neq i} \frac{1+\tau_{k}}{\left(1+2 \tau_{k}\right)^{2}} \\
& -\frac{2\left(3+2 \tau_{i}\right)\left(1+\tau_{i}\right)}{1+2 \tau_{i}}-2\left(3+2 \tau_{i}\right)\left(1+\tau_{i}\right) \sum_{k \neq i} \frac{1}{1+2 \tau_{k}}-2\left(3+2 \tau_{i}\right)\left(1+2 \tau_{i}\right) \sum_{k \neq i} \frac{1+2 \tau_{k}}{\left(1+2 \tau^{2}\right.} \\
& -2\left(3+2 \tau_{i}\right)\left(1+2 \tau_{i}\right)^{2} \sum_{k \neq i} \frac{1+\tau_{k}}{\left(1+2 \tau_{k}\right)^{2}} \sum_{k \neq i} \frac{1}{1+2 \tau_{k}}, \\
= & -2\left(1+\tau_{i}\right)^{2}-\left(3+2 \tau_{i}\right)\left(1+\tau_{i}\right) \sum_{k \neq i} \frac{1}{1+2 \tau_{k}}-8\left(1+2 \tau_{i}\right)\left(1+\tau_{i}\right)^{2} \sum_{k \neq i} \frac{1+\tau_{k}}{\left(1+2 \tau_{k}\right)^{2}} \\
& -2\left(3+2 \tau_{i}\right)\left(1+2 \tau_{i}\right)^{2} \sum_{k \neq i} \frac{1+\tau_{k}}{\left(1+2 \tau_{k}\right)^{2}} \sum_{k \neq i} \frac{1}{1+2 \tau_{k}} .
\end{aligned}
$$

$h_{i}(\tau)<0 \Longrightarrow \pi_{i}^{*}(\sigma)$ is decreasing in $\tau_{i}=\frac{\sigma_{i}^{2}}{\sigma_{v}^{2}}$.

$$
\begin{aligned}
\bullet \frac{\partial \ln \pi^{*}}{\partial \tau_{i}}(\tau) & =\frac{\partial}{\partial \tau_{i}}\left\{\frac{1}{2} \ln \left[\sum_{j=1}^{N} \frac{1+\tau_{j}}{\left(1+2 \tau_{j}\right)^{2}}\right]-\ln \left[1+\sum_{j=1}^{N} \frac{1}{1+2 \tau_{j}}\right]\right\} \\
& =-\frac{1}{2} \frac{3+2 \tau_{i}}{\left(1+2 \tau_{i}\right)^{3} \sum_{j=1}^{N} \frac{1+\tau_{j}}{\left(1+2 \tau_{j}\right)^{2}}}+\frac{2}{\left(1+2 \tau_{i}\right)^{2}\left[1+\sum_{j=1}^{N} \frac{1}{1+2 \tau_{j}}\right]},
\end{aligned}
$$




$$
=\frac{-\left(3+2 \tau_{i}\right)\left[1+\sum_{j=1}^{N} \frac{1}{1+2 \tau_{j}}\right]+4\left(1+2 \tau_{i}\right) \sum_{j=1}^{N} \frac{1+\tau_{j}}{\left(1+2 \tau_{j}\right)^{2}}}{2\left(1+2 \tau_{i}\right)^{3}\left[\sum_{j=1}^{N} \frac{1+\tau_{j}}{\left(1+2 \tau_{j}\right)^{2}}\right]\left[1+\sum_{j=1}^{N} \frac{1}{1+2 \tau_{j}}\right]},
$$

where:

$$
\begin{aligned}
h_{i}(\tau) & =-\left(3+2 \tau_{i}\right)-\frac{3+2 \tau_{i}}{1+2 \tau_{i}}-\left(3+2 \tau_{i}\right) \sum_{j \neq i} \frac{1}{1+2 \tau_{j}}+4 \frac{1+\tau_{i}}{1+2 \tau_{i}}+4\left(1+2 \tau_{i}\right) \sum_{j \neq i} \frac{1+\tau_{j}}{\left(1+2 \tau_{j}\right)^{2}} \\
& =-2\left(1+\tau_{i}\right)-\left(3+2 \tau_{i}\right) \sum_{j \neq i} \frac{1}{1+2 \tau_{j}}+4\left(1+2 \tau_{i}\right) \sum_{j \neq i} \frac{1+\tau_{j}}{\left(1+2 \tau_{j}\right)^{2}}, \\
& =-2 \tau_{i}\left(1+\sum_{j \neq i} \frac{1}{1+2 \tau_{j}}-4 \sum_{j \neq i} \frac{1+\tau_{j}}{\left(1+2 \tau_{j}\right)^{2}}\right)-2-3 \sum_{j \neq i} \frac{1}{1+2 \tau_{j}}+4 \sum_{j \neq i} \frac{1+\tau_{j}}{\left(1+2 \tau_{j}\right)^{2}}, \\
& =-2 \tau_{i}\left(1-\sum_{j \neq i} \frac{3+2 \tau_{j}}{\left(1+2 \tau_{j}\right)^{2}}\right)-\left(2+\sum_{j \neq i} \frac{2 \tau_{j}-1}{\left(1+2 \tau_{j}\right)^{2}}\right) \\
& =-\left[2 \tau_{i} b\left(\tau_{-i}\right)+c\left(\tau_{-i}\right)\right] .
\end{aligned}
$$

This ends the proof of proposition 4.3.

\section{B.4. Proof of proposition 4.4}

In order to show the result of the non-emptiness of $\Sigma_{N}$ for $N \geq 4$, we just need to focus on the symmetric case where $\sigma_{1}=\ldots=\sigma_{N}=\sigma$ and this is the purpose of proposition 5.2. We now show that $\Sigma_{N}$ is empty for $N \leq 3$.

Proof for $\mathrm{N}=2$

$$
\pi_{i}(\tau)=\sigma_{u} \sigma_{v} \frac{\frac{1+\tau_{i}}{\left(1+2 \tau_{i}\right)^{2}}}{\left\{\sum_{j=1}^{2} \frac{1+\tau_{j}}{\left(1+2 \tau_{j}\right)^{2}}\right\}^{\frac{1}{2}}\left\{1+\sum_{i=1}^{2} \frac{1}{1+2 \tau_{i}}\right\}} .
$$

We denote $x_{i}=\frac{1+\tau_{i}}{\left(1+2 \tau_{i}\right)^{2}}, i=1,2$. For $\tau_{i} \neq 0, x_{i}<1$ since $x_{i}=\frac{1}{1+2 \tau_{i}}-\frac{\tau_{i}}{\left(1+2 \tau_{i}\right)^{2}}$. 
$y_{i}=\frac{1}{1+2 \tau_{i}}=\frac{\sqrt{1+8 x_{i}}-1}{2}$ indeed:

$$
x_{i}=\frac{1}{2} y_{i}+\frac{1}{2} y_{i}^{2} \Longrightarrow \begin{aligned}
& y_{i}^{2}+y_{i}-2 x_{i}=0, \\
& y_{i}=\frac{\sqrt{1+8 x_{i}}-1}{2}>0 .
\end{aligned}
$$

Therefore we have $i=1,2$ :

$$
\begin{aligned}
\pi_{i}(\tau) & =\sigma_{v} \sigma_{u} \frac{2 x_{i}}{\left(\sum_{j=1}^{2} x_{j}\right)^{\frac{1}{2}}\left(\sum_{j=1}^{2} \sqrt{1+8 x_{j}}\right)} \\
\pi_{i}(0) & =\frac{\sigma_{v} \sigma_{u}}{3 \sqrt{2}}
\end{aligned}
$$

We already know that, for $\tau_{1}=\tau_{2} \neq 0, \pi_{1}(\tau)$ and $\pi_{2}(\tau)$ are strictly smaller than $\pi_{i}(0)=\frac{\sigma_{v} \sigma_{u}}{3 \sqrt{2}}$ and equal if and only if $\tau_{1}=\tau_{2}=0$. We thus focus on cases where $\tau_{1} \neq \tau_{2} \Longleftrightarrow x_{1} \neq x_{2}$. Suppose now (without any loss of generality) that $x_{1}>x_{2}$ and that $\pi_{i}(\tau) \geq \frac{\sigma_{v} \sigma_{u}}{3 \sqrt{2}}, i=1,2$.

$$
\Longrightarrow x_{i} \geq \frac{1}{6 \sqrt{2}}\left(\sum_{j=1}^{2} x_{j}\right)^{\frac{1}{2}}\left(\sum_{j=1}^{2} \sqrt{1+8 x_{j}}\right), i=1,2
$$

since $x_{1}>x_{2}$ :

$$
\begin{aligned}
& \Longrightarrow \quad x_{2} \geq \frac{1}{3} \sqrt{x_{2}} \sqrt{1+8 x_{2}}, \\
& \Longrightarrow \quad \sqrt{x_{2}} \geq \frac{1}{3} \sqrt{1+8 x_{2}}, \\
& \Longrightarrow \quad x_{2} \geq \frac{1}{9}\left(1+8 x_{2}\right), \\
& \Longrightarrow \quad x_{2} \geq 1,
\end{aligned}
$$

which is ruled out. This implies that $\Sigma_{2}$ is empty.

Proof for $\mathrm{N}=3$

$$
\pi_{i}(\tau)=\sigma_{u} \sigma_{v} \frac{\frac{1+\tau_{i}}{\left(1+2 \tau_{i}\right)^{2}}}{\left\{\sum_{j=1}^{3} \frac{1+\tau_{j}}{\left(1+2 \tau_{j}\right)^{2}}\right\}^{\frac{1}{2}}\left\{1+\sum_{i=1}^{3} \frac{1}{1+2 \tau_{i}}\right\}} .
$$

We again denote $x_{i}=\frac{1+\tau_{i}}{\left(1+2 \tau_{i}\right)^{2}}, i=1,2,3$. For $\tau_{i} \neq 0, x_{i}<1, y_{i}=\frac{1}{1+2 \tau_{i}}=\frac{\sqrt{1+8 x_{i}}-1}{2}$. 
Therefore we have $i=1,2,3$ :

$$
\begin{aligned}
& \pi_{i}(\tau)=\sigma_{v} \sigma_{u} \frac{2 x_{i}}{\left(\sum_{j=1}^{3} x_{j}\right)^{\frac{1}{2}}\left(\sum_{j=1}^{3} \sqrt{1+8 x_{j}}-1\right)} \\
& \pi_{i}(0)=\frac{\sigma_{v} \sigma_{u}}{4 \sqrt{3}}
\end{aligned}
$$

We already know that, for $\tau_{1}=\tau_{2}=\tau_{3} \neq 0, \pi_{1}(\tau), \pi_{2}(\tau)$ and $\pi_{3}(\tau)$ are strictly smaller than $\pi_{i}(0)=\frac{\sigma_{v} \sigma_{u}}{4 \sqrt{3}}$ and equal if and only if $\tau_{1}=\tau_{2}=\tau_{3}=0$. We thus focus on cases where $\exists(i, j) \in\{1,2,3\}^{2}$ such that $\tau_{j} \neq \tau_{i} \Longleftrightarrow x_{j} \neq x_{i}$. Without any loss of generality (since it corresponds to the generic case), we will suppose (up to a reordering) that:
a) either $x_{1}>x_{2} \geq x_{3}$,
b) or $x_{1} \geq x_{2}>x_{3}$.

Now assume that $\pi_{i}(\tau) \geq \frac{\sigma_{v} \sigma_{u}}{4 \sqrt{3}}, i=1,2,3$.

a) $x_{1}>x_{2} \geq x_{3}$ :

$$
\begin{aligned}
& \Longrightarrow \quad x_{3} \geq \frac{1}{8 \sqrt{3}}\left(\sum_{j=1}^{3} x_{j}\right)^{\frac{1}{2}}\left(\sum_{j=1}^{3} \sqrt{1+8 x_{j}}-1\right), \\
& \Longrightarrow \quad x_{3} \geq \frac{1}{8} \sqrt{x_{3}}\left(3 \sqrt{1+8 x_{3}}-1\right), \\
& \Longrightarrow 8 \sqrt{x_{3}} \geq 3 \sqrt{1+8 x_{3}}-1 .
\end{aligned}
$$

Let $f$ be:

$$
\begin{aligned}
& f(x)=8 \sqrt{x}-3 \sqrt{1+8 x}+1 \\
& 0 \leq x \\
& f^{\prime}(x)=\frac{4(1-x)}{\sqrt{x} \sqrt{1+8 x}[\sqrt{1+8 x}+3 \sqrt{x}]} .
\end{aligned}
$$

$f$ is increasing on $[0,1]$, decreasing on $[1,+\infty[$ and $f(1)=0$. Therefore $\forall x \geq 0, f(x) \leq 0$ and $f(x)=0 \Longrightarrow x=1$.

$$
8 \sqrt{x_{3}} \geq 3 \sqrt{1+8 x_{3}}-1 \Longrightarrow x_{3}=1
$$

which is ruled out.

b) $x_{1} \geq x_{2}>x_{3}$ : the same proof is available.

This implies that $\Sigma_{3}$ is empty.

\section{C.1. Proof of proposition 5.2}




$$
\begin{array}{ll}
N \geq 4, \quad \pi_{i}^{*}(\sigma)>\pi_{i}^{*}(0), \\
\Longleftrightarrow \quad \frac{\sqrt{1+\tau}}{\sqrt{N}(N+1+2 \tau)}>\frac{1}{\sqrt{N}(N+1)}, \quad \text { where } \tau=\frac{\sigma^{2}}{\sigma_{v}^{2}}, \\
\Longleftrightarrow \quad \sqrt{1+\tau}>1+\frac{2}{N+1} \tau, \\
\Longleftrightarrow \quad 1+\tau>1+\frac{4}{N+1} \tau+\frac{4}{(N+1)^{2}} \tau^{2}, \\
\Longleftrightarrow \quad\left(1-\frac{4}{N+1}\right) \tau>\frac{4}{(N+1)^{2}} \tau^{2}, \quad \tau>0, \\
\Longleftrightarrow \quad \tau<\frac{(N-3)(N+1)}{4}, \\
\Longleftrightarrow \quad \sigma<\frac{\sigma_{v}}{2} \sqrt{N-3} \sqrt{N+1} .
\end{array}
$$

This ends the proof of proposition 5.2 and by the way of proposition 4.4.

\section{C.2. Proof of proposition 5.4}

$$
\left(\begin{array}{c}
\widetilde{v} \\
\widetilde{w}
\end{array}\right) \leadsto \mathcal{N}\left[\left(\begin{array}{l}
0 \\
0
\end{array}\right),\left(\begin{array}{cc}
\sigma_{v}^{2} & \frac{\sqrt{N} \sigma_{u} \sigma_{v}}{\sqrt{1+\frac{\sigma^{2}}{\sigma_{v}^{2}}}} \\
\frac{\sqrt{N} \sigma_{u} \sigma_{v}}{\sqrt{1+\frac{\sigma^{2}}{\sigma_{v}^{2}}}} & \sigma_{u}^{2}\left[1+\frac{N+\frac{\sigma^{2}}{\sigma_{v}^{2}}}{1+\frac{\sigma^{2}}{\sigma_{v}^{2}}}\right]
\end{array}\right)\right],
$$

$\left(\begin{array}{c}\widetilde{v} \\ \widetilde{u}\end{array}\right)$ is normal $\Longrightarrow\left(\begin{array}{c}\widetilde{v} \\ \widetilde{w}\end{array}\right)$ is normal. Moreover we have:

- $\operatorname{Cov}(\widetilde{v}, \widetilde{w})=\operatorname{Cov}\left(\widetilde{v}, N \beta^{*}(\sigma, N) \widetilde{v}+\sum_{i=1}^{N} \beta^{*}(\sigma, N) \widetilde{\varepsilon} i+\widetilde{u}\right)=N \beta^{*}(\sigma, N) \sigma_{v}^{2}=\frac{\sqrt{N} \sigma_{u} \sigma_{v}}{\sqrt{1+\frac{\sigma^{2}}{\sigma_{v}^{2}}}}$. 
- $\operatorname{Var}(\widetilde{w})=\operatorname{Var}\left(N \beta^{*}(\sigma, N) \widetilde{v}+\sum_{i=1}^{N} \beta^{*}(\sigma, N) \widetilde{\varepsilon}_{i}+\widetilde{u}\right)=N^{2} \beta^{*^{2}}(\sigma, N) \sigma_{v}^{2}+N \beta^{*^{2}}(\sigma, N) \sigma^{2}+\sigma_{u}^{2}$, $\operatorname{Var}(\widetilde{w})=\frac{N \sigma_{u}^{2}}{1+\frac{\sigma^{2}}{\sigma_{v}^{2}}}+\frac{\sigma_{u}^{2} \frac{\sigma^{2}}{\sigma_{v}^{2}}}{1+\frac{\sigma^{2}}{\sigma_{v}^{2}}}+\sigma_{u}^{2}=\sigma_{u}^{2}\left[1+\frac{N+\frac{\sigma^{2}}{\sigma_{v}^{2}}}{1+\frac{\sigma^{2}}{\sigma_{v}^{2}}}\right]$

- $\operatorname{Var}(\widetilde{v} / w)=\operatorname{Var}(\widetilde{v})-\frac{[\operatorname{Cov}(\widetilde{v}, \widetilde{w})]^{2}}{\operatorname{Var}(\widetilde{w})}=\sigma_{v}^{2}\left[1-\frac{N}{N+1+2 \frac{\sigma^{2}}{\sigma_{v}^{2}}}\right]$.

This ends the proof of proposition 5.4.

\section{C.3. Proof of proposition 5.5}

$$
\begin{aligned}
\pi_{i}^{*}(\tau) & =\sigma_{v} \sigma_{u} \frac{\sqrt{1+\tau}}{\sqrt{N}(N+1+2 \tau)}, \quad \text { where } \tau=\frac{\sigma^{2}}{\sigma_{v}^{2}}, \\
\frac{\partial \ln \pi_{i}^{*}}{\partial \tau}(\tau) & =\frac{\partial}{\partial \tau}\left\{\frac{1}{2} \ln (1+\tau)-\ln (N+1+2 \tau)\right\} \\
& =\frac{1}{2(1+\tau)}-\frac{2}{N+1+2 \tau}=\frac{N-3-2 \tau}{2(1+\tau)(N+1+2 \tau)} .
\end{aligned}
$$

Therefore $\pi_{i}^{*}(\tau)$ is maximized for $\tau=\frac{N-3}{2}$ and this ends the proof of proposition 5.5. 